IMA Journal of Applied Mathematics Page 1 of 35

\title{
Nonlinear Waves in Lattices: Past, Present, Future
}

\author{
P.G. KEVREKIDIS \\ Department of Mathematics and Statistics, University of Massachusetts,
}

Amherst, MA 01003, USA.

\begin{abstract}
In the present work, we attempt a brief summary of various areas where nonlinear waves have been emerging in the phenomenology of lattice dynamical systems. These areas include nonlinear optics, atomic physics, mechanical systems, electrical lattices, nonlinear metamaterials, plasma dynamics and granular crystals. We give some of the recent developments in each one of these areas and speculate on some of the potentially interesting directions for future study.
\end{abstract}

Keywords:

\section{Introduction}

Over the last two decades, there has been an explosion of interest on the dynamics of nonlinear waves in lattices. It can be argued that this field was actually initiated by the seminal investigation of Fermi, Pasta and Ulam (FPU) presented in Fermi et al. (1954), who posed the following question: How long does it take for long-wavelength oscillations to transfer their energy into an equilibrium distribution in a one-dimensional string of nonlinearly interacting particles? This question spurred the activity of a wide array of fields including soliton theory, discrete lattice dynamics and KAM theory, all of which remain active research fields today.

The development of soliton theory, while focusing at the continuum level at first through the investigations of Zabusky \& Kruskal (1967) and the concomitant developments of the theory of integrability for the Korteweg-de Vries equation, also offered some of the first particularly interesting examples of lattice nonlinear dynamical systems. Among the prime examples thereof, one can classify the Toda lattice of Toda (1981), the Ablowitz-Ladik equation of Ablowitz \& Ladik (1976) and the Calogero-Moser $N$-body problem, see e.g. the works of Calogero (1971) and Moser (1975).

It was at around the same time that some of the early fundamental suggestions of the role of discrete solitons in physical systems were made in the 1970s. Some of the pioneers of these contributions were Davydov, see e.g. Davydov (1972) and Heeger and collaborators, see e.g. Su et al. (1979) and Heeger et al. (1988). Davydov proposed the discrete soliton as a tool for understanding energy transfer in proteins and their $\alpha$-helices, while Heeger and collaborators put forth solitonic models for understanding neutral and charge transport in conducting polymer chains such as polyacetylene and polythiophene.

However, a true explosion of the field came about in the late 1980s when not only major developments arose in the context of the so-called discrete self-trapping equation of Eilbeck et al. (1985), but also the work of Sievers \& Takeno (1988) and Takeno et al. (1988) brought forth the theme of intrinsic localized modes (or discrete breathers) in anharmonic lattices. Although this theme had been explored earlier e.g., by Ovchinnikov (1970), Kosevich \& Kovalev (1974) and Dolgov (1986), it was Sievers \& Takeno (1988) and Takeno et al. (1988) that motivated a number of other researchers to explore the existence and stability of such modes more thoroughly and to start obtaining fundamental results about their properties, as well as to devise special limits (such as the anti-continuum (AC) limit of lattices with uncoupled sites) which could be used to showcase the generic nature of these modes. 
A landmark in connection to these efforts was the work of MacKay \& Aubry (1994) which established rigorously the existence/robustness of such localized modes starting from the AC limit, under minimal appropriate (non-resonance) assumptions for chains of nonlinear oscillators.

Since these early steps, there has been a tremendous amount of developments in the area of nonlinear waves in dynamical lattices. This activity has chiefly been fueled by the experimental observation of these modes in a wide range of physical systems in biophysics, solid state physics, nonlinear optics, atomic physics, granular crystals, and plasma physics, in addition to numerous developments in the more classical fields of mechanical and electrical lattices [and even the latter have seen interesting recent developments e.g. with the emergence of the field of nonlinear metamaterials, among others, as discussed in section 4.2 below]. A (partial) list of key physical systems where explanations in terms of nonlinear waves in lattices have had an impact includes:

- The observation of discrete breathers in complex electronic materials such as halide-bridged transition metal complexes as e.g. in Swanson et al. (1999).

- The formation of denaturation bubbles in the DNA double strand dynamics summarized e.g. in Peyrard (2004).

- The emergence of dynamical instabilities, discrete quantum self-trapping and localized modes in ultracold Bose-Einstein condensates in the presence of deep optical lattices, see e.g. the reviews of Brazhnyi \& Konotop (2004) and Morsch \& Oberthaler (2006).

- The illustration of localized modes of solitonic, vortex, ring, multipole, surface, gap, necklace and numerous other types in the nonlinear optics of evanescently coupled waveguide arrays, as well as in that of biased photorefractive crystals; a relevant recent review can be found in Lederer et al. (2008).

- The prototypical mechanical realization of solitary waves and localized modes in driven micromechanical cantilever arrays as shown in Sato et al. (2006), as well as in coupled torsion pendula where a recent example is given by Cuevas et al. (2009).

- The examination of interesting properties and mobility of localized modes in nonlinear electrical lattices and transmission lines; see e.g. Remoissenet (1999) and English et al. (2008).

- The presence of nearly compact solitary waves in granular crystals consisting of beads with Hertzian elastic interactions reviewed in Nesterenko (2001) and Sen et al. (2008).

- The creation of discrete breather type structures in layered antiferromagnetic samples such as those of a $\left(\mathrm{C}_{2} \mathrm{H}_{5} \mathrm{NH}_{3}\right)_{2} \mathrm{CuCl}_{4}$ crystal, upon imposition of a dynamically unstable continuous wave (which falls into modulational instability and results in the localized waveforms); see the relevant works of English et al. (2001) and English et al. (2003).

These experimental developments have, in turn, motivated a huge range of theoretical advances in connection to the properties and interactions of these lattice nonlinear waves and on how these are modified in comparison to their continuum siblings. In particular, some of the areas that have seen intense theoretical activity concern:

- The existence and stability of the localized modes.

- The dynamics and interactions of multiple such states in nonlinear lattices. 
- The effect of external potentials (either local "impurity-like" potentials, or more broad externally imposed, field potentials)

- The effect of short-range (local) versus long-range (nonlocal) interactions.

- The presence of excitation thresholds for such nonlinear states (especially in higher dimensional settings).

- The statistical mechanics and long-time asymptotics of these lattice systems and the role of the localized modes in them.

- The effects of disorder and the role of the interplay between the linear phenomenon of Anderson localization and of the nonlinearity-induced type of localization.

- The interaction with and scattering of phonon-like modes from discrete solitary waves.

- The modifications imparted on the waveforms of such localized states and the new possibilities for ones such (e.g. with embedded vorticity) afforded by higher dimensional settings.

Many of these topics have been summarized in a number of reviews by Braun \& Kivshar (1998), Aubry (1997), Flach \& Willis (1998), Hennig \& Tsironis 1998), Kevrekidis et al. (2001), Eilbeck \& Johansson (2003), Flach \& Gorbach (2008) and more recently in a number of books e.g. about Klein-Gordon nonlinear lattices by Braun \& Kivshar (2006), as well as about nonlinear Schrödinger ones by Kevrekidis (2009a).

As it can be inferred from the shear volume of review articles on the topic (as well as that of fullblown books), it is impossible to give an exhaustive description of any particular aspect or application of the subject in anything less than a lengthy review or a book. Our aim herein will thus, by necessity, be more modest, but in some sense, also more "forward looking". We aim to give a selective presentation of some of recent developments of a few among these areas, which in the present author's evaluation hold significant potential for future developments in the field of localized modes, nonlinear waves and discrete breathers. Obviously, this bears a significant weight of subjective perception and a personalized viewpoint about which we caution the reader in advance. On the other hand, our hope is to offer a number of interesting directions for future study in these areas which could significantly enhance our understanding of nonlinear waves in these settings, promoting their state-of-the-art and offering significant connections with present or near-future experimental settings and potentially also with applications.

We will categorize our presentation based on the different models and the corresponding areas of interest. Hence, upon introducing our basic notation, the third section will concern itself with localized modes in models of the discrete nonlinear Schrödinger type and their applications in nonlinear optics and atomic physics. The fourth section will consider models of the Klein-Gordon type, as well as their mechanical and electrical (as well as nonlinear metamaterial) applications. The fifth section will focus on models of the FPU type arising in the examination of granular crystals with Hertzian (or modified Hertzian) interactions, as well as in the case of study of dusty plasmas. Lastly, in section six, we will summarize some of our discussion and offer some concluding thoughts.

\section{Notation}

The prototypical dynamical lattices that will be considered in this review will be the discrete nonlinear Schrödinger equation (DNLS) in the form:

$$
i \dot{u}_{n}=-\varepsilon\left(u_{n+1}+u_{n-1}\right)-\left|u_{n}\right|^{2} u_{n},
$$


the nonlinear Klein-Gordon (KG) lattice of the form:

$$
\ddot{u}_{n}=\varepsilon\left(u_{n+1}+u_{n-1}-2 u_{n}\right)-V^{\prime}\left(u_{n}\right),
$$

and the FPU-type dynamical lattice of the form

$$
\ddot{u}_{n}=V^{\prime}\left(u_{n+1}-u_{n}\right)-V^{\prime}\left(u_{n}-u_{n-1}\right),
$$

where $u_{n}$ is the dynamical variable of interest measured at site $n, \varepsilon$ is the coupling parameter and $V$ a potential (that could be onsite for KG lattices or inter-site for FPU ones).

It should be noted that in what follows, we will not restrict ourselves to the one-dimensional framework of Eqs. (2.1)- (2.3), but motivated by physical applications, we will often consider higher dimensional variants of the models. In their prototypical form, two-dimensional generalizations of Eqs. (2.1)- (2.3) read

$$
i \dot{u}_{n, m}=-\varepsilon\left(u_{n+1, m}+u_{n-1, m}+u_{n, m+1}+u_{n, m-1}\right)-\left|u_{n, m}\right|^{2} u_{n, m},
$$

for the DNLS lattice,

$$
\ddot{u}_{n, m}=\varepsilon\left(u_{n+1, m}+u_{n-1, m}+u_{n, m+1}+u_{n, m-1}-4 u_{n, m}\right)-V^{\prime}\left(u_{n, m}\right),
$$

for the KG case, while for the FPU-type lattices we have

$$
\ddot{u}_{n, m}=V^{\prime}\left(u_{n+1, m}-u_{n, m}\right)-V^{\prime}\left(u_{n, m}-u_{n-1, m}\right)+V^{\prime}\left(u_{n, m+1}-u_{n, m}\right)-V^{\prime}\left(u_{n, m}-u_{n, m-1}\right) .
$$

This, in turn, clearly suggests how to expand the model beyond two dimensions and into the three- or arbitrary-dimensional-case, by sequentially adding more lattice directions and linear (in cases such as (2.4)-(2.5) or nonlinear (as in case (2.6) interactions along them.

In the first one of these, the field will be complex and we will most often seek stationary (standing wave) type solutions of the form $u_{n}=\exp (i \mu t) v_{n} ; \mu$ is often referred to as the propagation constant in nonlinear optics, or the chemical potential in Bose-Einstein condensates and characterizes the frequency of such standing wave solutions. These will satisfy the steady state equation:

$$
\left(\mu-\left|v_{n}\right|^{2}\right) v_{n}=\varepsilon\left(v_{n+1}+v_{n-1}\right) .
$$

Additionally, one of the fundamental ideas that we will use in exploring this class of systems will be that of the anti-continuum limit of MacKay \& Aubry (1994). This is the limit where the sites are uncoupled with $\varepsilon=0$. In this case, Eq. (2.7) is completely solvable $v_{n}=0, \pm \sqrt{\mu} \exp \left(i \theta_{n}\right)$, where $\theta_{n}$ is a free phase parameter for each site. The fundamental question of interest will involve the persistence of these solutions for $\varepsilon \neq 0$, as well as their stability. The latter will be often explored by means of linear stability analysis, upon imposition of a perturbation of the form:

$$
u_{n}(t)=\exp (i \mu t)\left[v_{n}+\delta\left(p_{n} \exp (\lambda t)+q_{n}^{\star} \exp \left(\lambda^{\star} t\right)\right)\right] .
$$

[Notice that the ${ }^{\star}$ will be generally used in what follows to denote complex conjugation.] Consideration of the resulting equation upon substitution of $(2.8)$ to $\sqrt{2.1]}$, at $\mathscr{O}(\delta)$ yields the linearization (infinitedimensional matrix) eigenvalue problem for the eigenvalues $\lambda$ and eigenvectors $\left(p_{n}, q_{n}\right)$.

In the case of the Klein-Gordon models, we will focus on discrete breather time-periodic solutions for different potentials which are either polynomials in the form $V\left(u_{n}\right)=\sum_{n=2}^{N} a_{j} u_{n}^{j}$ or other functions such as $V\left(u_{n}\right)=1-\cos \left(u_{n}\right)$ (in the sine-Gordon case). Notice also that we will often use the operator 
notation $\Delta_{2} u_{n}=u_{n+1}+u_{n-1}-2 u_{n}$ for the discrete Laplacian. For the FPU lattices, we will primarily concern ourselves with power law potentials and particularly the Hertzian elastic case of $V\left(r_{n}\right) \sim r_{n}^{5 / 2}$, but we will also briefly refer to other potentials such as the Yukawa one $V\left(r_{n}\right) \sim \exp \left(-\alpha r_{n}\right) / r_{n}$ (for $r_{n}>0$ ) in the context of dusty plasmas. Notice that in the FPU-type chains, we will also refer to the so-called strain formulation which introduces the modified field variable $r_{n}=u_{n-1}-u_{n}$ and leads to the dynamical equation

$$
\ddot{r}_{n}=V^{\prime}\left(r_{n+1}\right)+V^{\prime}\left(r_{n-1}\right)-2 V^{\prime}\left(r_{n}\right) .
$$

\section{DNLS Models in Nonlinear Optics and Atomic Physics}

\subsection{Recent Developments}

Many of the investigations in these fields stemmed from the relevance of the DNLS model and its variants in the nonlinear optics of fabricated AlGaAs waveguide arrays as in Eisenberg et al. (1998), as well as in that of Bose-Einstein condensates in sufficiently deep optical lattices analyzed in Brazhnyi \& Konotop (2004), Morsch \& Oberthaler (2006), and Kevrekidis et al. (2008a).

In the former area, a multiplicity of phenomena such as discrete diffraction, Peierls barriers (the energetic barrier that a wave needs to overcome to move from one lattice site to the next), diffraction management (the periodic alternation of the diffraction coefficient) in Morandotti et al. (1999) and gap solitons (structures localized due to nonlinearity in the gap of the underlying linear spectrum) in Mandelik et al. (2004) among others [see also Morandotti et al. (1999)] were experimentally observed. More recently, fundamental investigations also on the modulational instability arising in such settings for simple plane wave solutions in Meier et al. (2004), and on the role of multiple components and possible four-wave-mixing effects arising from their coupling in Meier et al. (2003) have been explored, as well as that of interactions of solitary waves with surfaces in Suntsov et al. (2006).

A related area where lattice dynamical models, although not directly relevant as the most appropriate description (since the setting is, in principle continuum with a periodic potential), still yield accurate predictions regarding the existence and the stability of nonlinear localized modes is that of optically induced lattices in photorefractive media such as Strontium Barium Niobate (SBN). Since the theoretical suggestion of such lattices in Efremidis et al. (2002), and their experimental realization in Fleischer et al. (2003), Neshev et al. (2003) and Martin et al. (2004), there has been a tremendous growth in the area of nonlinear waves and solitons in such periodic, predominantly two-dimensional, lattices. The continuously growing array of structures that have been predicted and experimentally obtained in such lattices includes (but is not limited to) various discrete multi-pulse patterns, such as the discrete dipole solitons in Yang et al. (2004), quadrupole solitons in Yang et al. (2004), necklace solitons in Yang et al. (2005), soliton stripes in Neshev et al. (2004), discrete vortices in Neshev et al. (2004), and Fleischer et al. (2004), rotary solitons in Wang et al. (2006), surface solitons in Wang et al. (2007), and so on. Such structures have a definite potential to be used as carriers and conduits for data transmission and processing, in the setting of all-optical communication schemes; see a detailed recent review in Lederer et al. (2008).

Lastly, a completely independent and entirely different field where relevant considerations and structures, as well as models of the DNLS type arise is that of ultracold atomic gases in the form of BoseEinstein condensates (BECs) under the confinement imposed by so-called optical lattice potentials. These are periodic traps produced by counter-propagating laser beams in one, two or even all three directions, see e.g. Burger et al. (2005). This field has also experienced a tremendous amount of development in the past few years with some of its landmarks being the prediction [in Smerzi et al. (2002)] and 
manifestation [in Cataliotti et al. (2003)] of modulational instabilities, the observation of gap solitons in Eiermann et al. (2004), of Landau-Zener tunneling (tunneling between different bands of the periodic potential) in Jona-Lasinio et al. (2003) and Bloch oscillations (for matter waves subject to combined periodic and linear potentials) in Anderson \& Kasevich (1998) among many other salient features.

All of the above settings are described at some level of approximation by a continuum nonlinear Schrödinger (NLS) equation with a periodic potential. However, when the periodic potential is sufficiently deep, it was realized that an equivalent description of the system can be given by a nonlinear dynamical lattice as discussed e.g. in Trombettoni \& Smerzi (2001), and Abdullaev et al. (2001). This was further elaborated for BEC settings in Alfimov et al. (2002) [see also Ablowitz et al. (2004) for a related discussion in connection to nonlinear optics], whose presentation we briefly follow here.

The localized wavefunctions at the wells of the periodic potential can be approximated by Wannier functions, i.e., the Fourier transform of Bloch functions. Given the completeness of the Wannier basis, any solution of the NLS equation with a periodic potential can be expressed as $u(x, t)=$ $\sum_{n, \alpha} c_{n, \alpha}(t) w_{n, \alpha}(z)$, where $n$ and $\alpha$ label wells and bands of the periodic potential, respectively.

Substituting the above expression into the dynamical equation, and using the orthonormality of the Wannier basis as indicated in Alfimov et al. (2002), we obtain a set of differential equations for the coefficients. Upon suitable decay of the Fourier coefficients and the Wannier functions' prefactors (which can be systematically checked for given potential parameters), the model can be reduced to

$$
i \frac{d c_{n, \alpha}}{d t}=\hat{\omega}_{0, \alpha} c_{n, \alpha}+\hat{\omega}_{1, \alpha}\left(c_{n-1, \alpha}+c_{n+1, \alpha}\right)+\sum_{\alpha_{1}, \alpha_{2}, \alpha_{3}} W_{\alpha \alpha_{1} \alpha_{2} \alpha_{3}}^{n n n n} c_{n, \alpha_{1}}^{*} c_{n, \alpha_{2}} c_{n, \alpha_{3}},
$$

where $W_{\alpha \alpha_{1} \alpha_{2} \alpha_{3}}^{n n_{1} n_{2} n_{3}}=\int_{-\infty}^{\infty} w_{n, \alpha} w_{n_{1}, \alpha_{1}} w_{n_{2}, \alpha_{2}} w_{n_{3}, \alpha_{3}} d x$ is the Wannier function overlap integral and

$$
\hat{\omega}_{n, \alpha}=\frac{L}{2 \pi} \int_{-\pi / L}^{\pi / L} E_{\alpha}(k) e^{-i k n L} d k,
$$

is the Fourier transform of the energy $E_{\alpha}(k)$ of the Bloch mode corresponding to wavenumber $k$ for a potential of spatial period $L$. This degenerates into the so-called tight-binding model

$$
i \frac{d c_{n, \alpha}}{d t}=\hat{\omega}_{0, \alpha} c_{n, \alpha}+\hat{\omega}_{1, \alpha}\left(c_{n-1, \alpha}+c_{n+1, \alpha}\right)+W_{1111}^{n n n n}\left|c_{n, \alpha}\right|^{2} c_{n, \alpha},
$$

i.e., the DNLS equation, when restricting consideration to the first band. Higher-dimensional versions of the latter are of course physically relevant models and have, therefore, been used in various studies concerning quasi-2D and 3D BECs confined in strong optical lattices as illustrated in Kevrekidis et al. (2008a) and similarly in 2D waveguide arrays as summarized in Kevrekidis (2009a).

The relevance of the DNLS model in this wide array of application areas prompted an intense activity in investigating the fundamental solitary wave solutions of the model. We now focus on analyzing these prototypical solutions and their stability in the context of Eq. 2.1). In particular, for stationary solutions, upon multiplying Eq. (2.7) by $v_{n}^{*}$ and subtracting the complex conjugate of the resulting equation leads to:

$$
v_{n}^{*} v_{n+1}-v_{n} v_{n+1}^{*}=\text { const. } \Rightarrow 2 \arg \left(v_{n+1}\right)=2 \arg \left(v_{n}\right),
$$

for localized solutions (i.e., vanishing as $n \rightarrow \pm \infty$ ). Using the scaling freedom of the equation to set $\mu=$ 1 allows us to infer that the only states that will persist for finite $\varepsilon$ will be the ones containing sequences with combinations of $v_{n}= \pm 1$ and $v_{n}=0$. The work of Alfimov et al. (2004) provided a systematic numerical classification of the most fundamental of the resulting sequences and their bifurcations. Some 

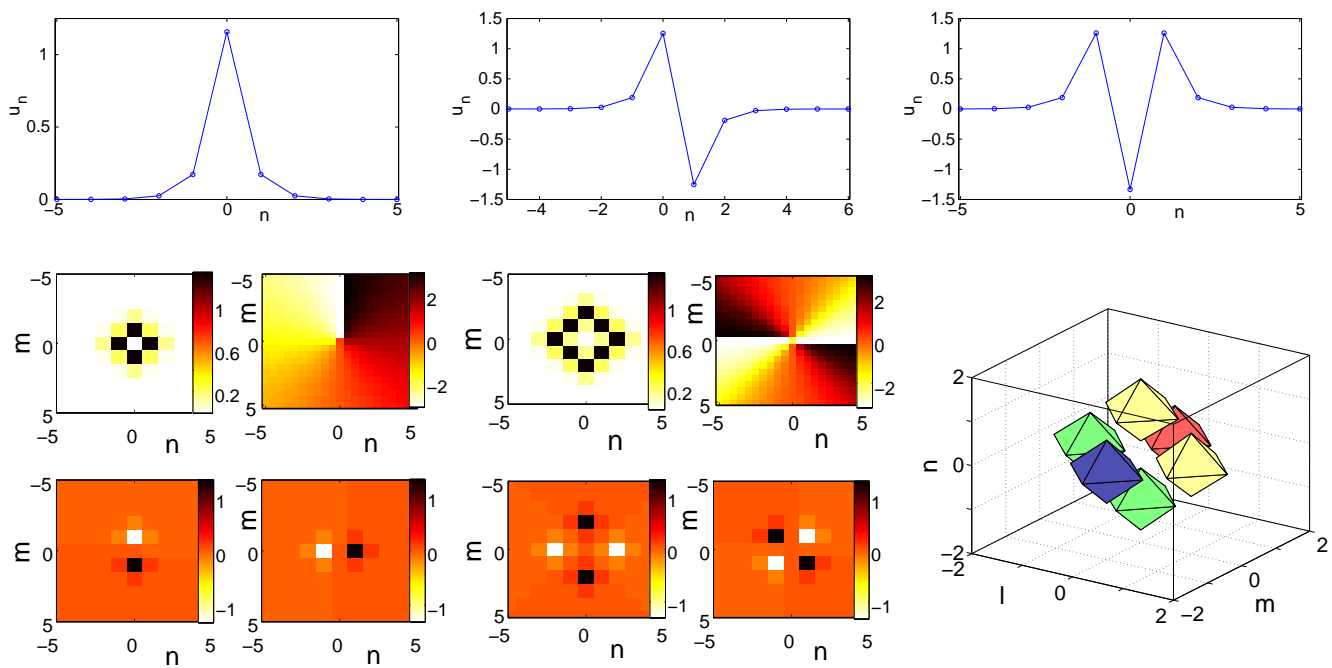

FIG. 1. (Color online) A sampler of solutions of the discrete nonlinear Schrödinger equation in 1-, 2- and 3-spatial lattice dimensions. The top left shows the always stable fundamental single soliton. The top middle and right show the out-of-phase two- and three-site excitations (which can be stable near the anti-continuum limit). The bottom left (4-panel set including the amplitude, phase, real and imaginary part contour plots of the solution) shows the prototypical vortex of topological charge $S=1$ (which is also stable near the AC limit). The bottom middle panel contains a rhombic $S=2$ generalization thereof with 8 sites (which may also be stable), while the bottom right contains the also stable near the AC limit 6-site diamond three-dimensional configuration.

of the prototypical examples of sequences involving one, two and three sites are illustrated in Fig. 1 While in our discussion below, we restrict our consideration to the case of focusing nonlinearity, our results can be extended to the defocusing case [with the opposite sign of the nonlinearity in Eq. (2.1)] via the so-called staggering transformation $w_{n}=(-1)^{n} u_{n}$. This converts the defocusing nonlinearity into a focusing one, with an appropriate frequency rescaling which can be trivially absorbed in a phase or gauge transformation.

We now turn to the examination of stability. Using the linearization ansatz of Eq. (2.8) and a simple eigenvector rotation leads to the equivalent symplectic formalism of the linear stability problem $J \mathscr{L} w=$ $\lambda w$, where $\mathscr{L}$ is a diagonal, self-adjoint matrix with the operators $\mathscr{L}_{+}$and $\mathscr{L}_{-}$given below in its diagonal entries and $J$ is the symplectic matrix. $\mathscr{L}_{+}$and $\mathscr{L}_{-}$are defined by:

$$
\begin{aligned}
\left(1-3 v_{n}^{2}\right) a_{n}-\varepsilon\left(a_{n+1}+a_{n-1}\right) & =\mathscr{L}_{+} a_{n}=-\lambda b_{n} \\
\left(1-v_{n}^{2}\right) b_{n}-\varepsilon\left(b_{n+1}+b_{n-1}\right) & =\mathscr{L}_{-} b_{n}=\lambda a_{n} .
\end{aligned}
$$

Using once again the AC limit, we assume a $v_{n}$ with $N$ "excited" (i.e., $\neq 0$ ) sites; it can then be straightforwardly inferred that for $\varepsilon=0$ these sites correspond to eigenvalues $\lambda_{+}=-2$ for $\mathscr{L}_{+}$and to ones with $\lambda_{-}=0$ for $\mathscr{L}_{-}$. In turn, these imply the existence of $N$ eigenvalue pairs with $\lambda^{2}=0$ for the full problem. These $N$ vanishing eigenvalue pairs are potential sources of instability, since $N-1$ of those will become nonzero, upon departure from the AC limit, given that there is a single symmetry left for nonzero $\varepsilon$, namely the $\mathrm{U}(1)$ invariance i.e., the invariance with respect to phase. The key issue for 
stability purposes is to identify the location of these $N-1$ small eigenvalue pairs. One can manipulate Eqs. 3.5-3.6 into the form:

$$
\mathscr{L}_{-} b_{n}=-\lambda^{2} \mathscr{L}_{+}^{-1} b_{n} \Rightarrow \lambda^{2}=-\frac{\left(b_{n}, \mathscr{L}_{-} b_{n}\right)}{\left(b_{n}, \mathscr{L}_{+}^{-1} b_{n}\right)} .
$$

In the vicinity of the $\mathrm{AC}$ limit, the effect of $\mathscr{L}_{+}$is a multiplicative one (by -2$)$. Hence:

$$
\lim _{\varepsilon \rightarrow 0}\left(b_{n}, \mathscr{L}_{+}^{-1} b_{n}\right)=-\frac{1}{2} \Rightarrow \lambda^{2}=2 \gamma=2\left(b_{n}, \mathscr{L}_{-} b_{n}\right) .
$$

In light of this calculation, the full problem becomes equivalent to the determination of the spectrum of $\mathscr{L}_{-}$. A crucial fact in that regard is that $v_{n}$ is an eigenfunction of $\mathscr{L}_{-}$with $\lambda_{-}=0$. Then, direct use of the Sturm comparison theorem for difference operators [see e.g. Levy \& Lessman (1992)] leads us to conclude that if the number of sign changes in the solution at the AC limit is $m$ (i.e., the number of times that adjacent to a +1 is a -1 and next to a -1 is a +1$)$, then $n\left(\mathscr{L}_{-}\right)=m$ and therefore from Eq. (3.8), the number of imaginary eigenvalues pairs of $J \mathscr{L}$ is $m$. This, in turn, implies that the number of real eigenvalue pairs is consequently $(N-1)-m$. Another immediate conclusion is that unless $m=N-1$, i.e., unless adjacent sites are out-of-phase with respect to each other, the solution will be immediately unstable upon departure from the AC limit. This analysis originally presented in Pelinovsky et al. (2005a) is also consistent with the general eigenvalue count of Kapitula et al. (2005). It should be noted that these $m$ imaginary eigenvalue pairs end up possessing, so-called, negative Krein signature as analyzed in Pelinovsky et al. (2005a). This implies that upon collision with other eigenvalue pairs, such as the ones stemming from the continuous spectrum, they will lead to instability through a socalled Hamiltonian-Hopf bifurcation and a complex eigenvalue quartet. In this problem, the continuous spectrum eigenmodes of eigenfrequency of wavenumber $k$ are characterized by the dispersion relation $\lambda= \pm i\left(1+4 \varepsilon \sin ^{2}(k / 2)\right)$, and hence correspond to a band of width $4 \varepsilon$, separated by a unit distance from the origin along the imaginary axis of the spectral plane $\left(\lambda_{r}, \lambda_{i}\right)$ of the eigenvalues $\lambda=\lambda_{r}+i \lambda_{i}$.

Equation (3.8) can also be used in a quantitative fashion to identify the relevant eigenvalues perturbatively for the full problem. In particular, this amounts to considering the eigenvalues of $\mathscr{L}_{-}$emanating from 0 through the perturbed eigenvalue problem:

$$
\mathscr{L}_{-}^{(0)} b_{n}^{(1)}=\gamma_{1} b_{n}^{(0)}-\mathscr{L}_{-}^{(1)} b_{n}^{(0)},
$$

where $\mathscr{L}_{-}=\mathscr{L}_{-}^{(0)}+\varepsilon \mathscr{L}_{-}^{(1)}+\mathscr{O}\left(\varepsilon^{2}\right)$ and a similar expansion has been used for the eigenvector $b_{n}$. For these eigenvalues, we know that $\lambda_{-}=\varepsilon \gamma_{1}+\mathscr{O}\left(\varepsilon^{2}\right)$. Projecting the above equation to all the eigenvectors of zero eigenvalue of $\mathscr{L}_{-}^{(0)}$, one can explicitly convert Eq. 3.9) into an eigenvalue problem of the form $M c=\gamma_{1} c$, as was shown in Pelinovsky et al. (2005a). The matrix $M$ has off-diagonal entries: $M_{n, n+1}=M_{n+1, n}=-\cos \left(\theta_{n+1}-\theta_{n}\right)$ and diagonal entries $M_{n, n}=\cos \left(\theta_{n-1}-\theta_{n}\right)+\cos \left(\theta_{n+1}-\theta_{n}\right)$. The subsequent computation of the leading order correction $\gamma_{1}$ (to the eigenvalues of $\mathscr{L}_{-}$) allows us to calculate the perturbed eigenvalues of the full problem $\lambda= \pm \sqrt{2 \varepsilon \gamma_{1}}$.

Let us consider as a case example the one-dimensional configurations with two-adjacent sites with phases $\theta_{1}$ and $\theta_{2}$. Then, the matrix $M$ becomes:

$$
M=\left(\begin{array}{cc}
\cos \left(\theta_{1}-\theta_{2}\right) & -\cos \left(\theta_{1}-\theta_{2}\right) \\
-\cos \left(\theta_{1}-\theta_{2}\right) & \cos \left(\theta_{1}-\theta_{2}\right)
\end{array}\right)
$$

which leads to $\lambda^{2}=0$ and $\lambda^{2}=4 \varepsilon \cos \left(\theta_{1}-\theta_{2}\right)$. Notice that, as expected, for same phase excitations $\left(\theta_{1}=\theta_{2}\right)$, the configuration is unstable due to a real eigenvalue pair, while the opposite is true if $\theta_{1}=$ 
$\theta_{2} \pm \pi$. This type of analysis is possible e.g. for 3 -site configurations with phases $\theta_{1,2,3}$ (or for that matter, for arbitrary numbers of excited sites). In the 3 -site case, one of the eigenvalues of $M$ is again 0 (this is true for any configuration due to the $\mathrm{U}(1)$ invariance), while the other two are given by:

$$
\begin{aligned}
\gamma_{1} & =\cos \left(\theta_{2}-\theta_{1}\right)+\cos \left(\theta_{3}-\theta_{2}\right) \\
& \pm \sqrt{\cos ^{2}\left(\theta_{2}-\theta_{1}\right)-\cos \left(\theta_{2}-\theta_{1}\right) \cos \left(\theta_{3}-\theta_{2}\right)+\cos ^{2}\left(\theta_{3}-\theta_{2}\right)}
\end{aligned}
$$

This formulation of the existence and stability problems has been generalized to different settings, such as higher dimensions in Pelinovsky et al. (2005b), and Pelinovsky et al. (2008) or multi-component systems analyzed e.g. in Kevrekidis \& Pelinovsky (2006). Arguably, the principal difference that arises in the higher dimensional settings is that the wave profile may contain sites excited over a contour (as opposed to along a straight line). In that case, for the $N$ excited sites around the contour, the persistence (Lyapunov-Schmidt) conditions can be obtained as a generalization of Eq. 2.1] that reads [see e.g. Pelinovsky et al. (2005b) and Alexander et al. (2004)]:

$$
\sin \left(\theta_{1}-\theta_{2}\right)=\sin \left(\theta_{2}-\theta_{3}\right)=\cdots=\sin \left(\theta_{N}-\theta_{1}\right) .
$$

This highlights a key difference of these higher dimensional settings, namely that not only "solitary wave" structures with phases $\theta \in\{0, \pi\}$ are possible, but also both symmetric and asymmetric vortex families presented in Pelinovsky et al. (2005b) and Alexander et al. (2004) may, in principle, exist [a note of caution, however, is that Eq. (3.12) provides only the leading order persistence condition and one would need to also verify the corresponding conditions to higher order to confirm that such solutions persist as was discussed in Pelinovsky et al. (2005b)]. These vortex solutions had been predicted numerically earlier in Malomed \& Kevrekidis (2001) and Kevrekidis et al. (2004a) and have been observed experimentally in the optical setting of photorefractive crystals by Neshev et al. (2004) and Fleischer et al. (2004). The stability analysis is also possible for these higher dimensional structures, although the relevant calculations are technically considerably more involved. In fact, these types of calculations are possible not only for square lattices, as in the cases highlighted above, but for more complex lattice settings as well including hexagonal and honeycomb lattices as shown in Law et al. (2008). A number of interesting conclusions arise therein including e.g. the instability of a lower topological charge (with vorticity $S=1$ ) vortex and the stability of a higher topological charge one (with $S=2$ ); such results have been recently also confirmed experimentally by Terhalle et al. (2009). Some case examples of interesting two-dimensional structures are given in Fig. 1 including a prototypical vortex cross of topological charge $S=1$, as well as a potentially stable $S=2$ generalization thereof in a rhombic configuration discussed recently in Öster \& Johansson (2006) and Law et al. (2009).

However, it should also be noted that the relevant theory can be formulated in an entirely general manner: we give the outline below, as well as illustrate some prototypical higher-dimensional (i.e., 3D) structures. In the multi-dimensional case, the problem of existence of stationary standing wave solutions can be formulated through the vanishing of the vector field $\mathbf{F}_{n}$ of the form:

$$
\mathbf{F}_{n}(\phi, \varepsilon)=\left[\begin{array}{c}
\left(1-\left|\phi_{n}\right|^{2}\right) \phi_{n}-\varepsilon \Sigma \phi_{n} \\
\left(1-\left|\phi_{n}\right|^{2}\right) \phi_{n}^{*}-\varepsilon \Sigma \phi_{n}^{*}
\end{array}\right]
$$


Upon defining the matrix operator:

$$
\begin{aligned}
\mathscr{H}_{n} & =\left(\begin{array}{cc}
1-2\left|\phi_{n}\right|^{2} & -\phi_{n}^{2} \\
-\phi_{n}^{* 2} & 1-2\left|\phi_{n}\right|^{2}
\end{array}\right) \\
& -\varepsilon\left(s_{+e_{1}}+s_{-e_{1}}+s_{+e_{2}}+s_{-e_{2}}+s_{+e_{3}}+s_{-e_{3}}\right)\left(\begin{array}{ll}
1 & 0 \\
0 & 1
\end{array}\right),
\end{aligned}
$$

where the $s_{ \pm e_{i}}$ denote the shift operators along the respective directions and $e_{i}$ are the corresponding unit vectors in each of the three lattice directions, the stability problem reads $\sigma \mathscr{H} \psi=i \lambda \psi$. Here each $2 \times 2$ block of the matrix $\sigma$ is the diagonal matrix with elements $(1,-1)$ along the diagonal for each node $n$; see Pelinovsky et al. (2008) for a detailed discussion. The existence problem, on the other hand, is connected to $\mathscr{H}$ through: $\mathscr{H}=D_{\phi} \mathbf{F}(\phi, 0)$. At the AC limit of $\varepsilon=0$

$$
\left(\mathscr{H}^{(0)}\right)_{n}=\left[\begin{array}{ll}
1 & 0 \\
0 & 1
\end{array}\right], n \in S^{\perp}, \quad\left(\mathscr{H}^{(0)}\right)_{n}=\left[\begin{array}{cc}
-1 & -e^{2 i \theta_{n}} \\
-e^{-2 i \theta_{n}} & -1
\end{array}\right], n \in S,
$$

where $S$ is the set of excited sites. Then the eigenvectors of zero eigenvalue will be of the form:

$$
\left(\mathbf{e}_{n}\right)_{k}=i\left[\begin{array}{c}
e^{i \theta_{n}} \\
-e^{-i \theta_{n}}
\end{array}\right] \delta_{k, n} .
$$

It is helpful to define the projection operator:

$$
(\mathscr{P} \mathbf{f})_{n}=\frac{\left(\mathbf{e}_{n}, \mathbf{f}\right)}{\left(\mathbf{e}_{n}, \mathbf{e}_{n}\right)}=\frac{1}{2 i}\left(e^{-i \theta_{n}}\left(\mathbf{f}_{1}\right)_{n}-e^{i \theta_{n}}\left(\mathbf{f}_{2}\right)_{n}\right), \quad n \in S,
$$

and to decompose the solution as:

$$
\phi=\phi^{(0)}(\theta)+\varphi \in X .
$$

Then, one can obtain the Lyapunov-Schmidt persistence conditions analyzed in Pelinovsky et al. (2008) as:

$$
\mathbf{g}(\theta, \varepsilon)=\mathscr{P} \mathbf{F}\left(\phi^{(0)}(\theta)+\varphi(\theta, \varepsilon), \varepsilon\right)=0 .
$$

As proved in Pelinovsky et al. (2008), the configuration $\phi^{(0)}(\theta)$ can then be continued to the domain $\varepsilon \in \mathscr{O}(0)$ (i.e., for couplings in the neighborhood of the AC limit) if and only if there exists a root $\theta_{*}$ of the vector field $\mathbf{g}(\theta, \varepsilon)$. Moreover, if the root $\theta_{*}$ is analytic in $\varepsilon \in \mathscr{O}(0)$ and $\theta_{*}=\theta_{0}+\mathscr{O}(\varepsilon)$, the solution $\phi$ of the difference equation is analytic in $\varepsilon \in \mathscr{O}(0)$, such that

$$
\phi=\phi^{(0)}\left(\theta_{*}\right)+\varphi\left(\theta_{*}, \varepsilon\right)=\phi^{(0)}\left(\theta_{0}\right)+\sum_{k=1}^{\infty} \varepsilon^{k} \phi^{(k)}\left(\theta_{0}\right) .
$$

Within the same formulation, one can establish a general stability theory, provided that the relevant solution persists for $\varepsilon \neq 0$. If the operator $\mathscr{H}$ has a small eigenvalue $\mu$ of multiplicity $d$, such that $\mu=\varepsilon^{k} \mu_{k}+\mathscr{O}\left(\varepsilon^{k+1}\right)$, then the full Hamiltonian eigenvalue problem admits $(2 d)$ small eigenvalues $\lambda$. These are such that $\lambda=\varepsilon^{k / 2} \lambda_{k / 2}+\mathscr{O}\left(\varepsilon^{k / 2+1}\right)$, where non-zero values $\lambda_{k / 2}$ are found from

$$
\begin{aligned}
& \text { odd } k: \quad \mathscr{M}^{(k)} \alpha=\frac{1}{2} \lambda_{k / 2}^{2} \alpha, \\
& \text { even } k: \quad \mathscr{M}^{(k)} \alpha+\frac{1}{2} \lambda_{k / 2} \mathscr{L}^{(k)} \alpha=\frac{1}{2} \lambda_{k / 2}^{2} \alpha,
\end{aligned}
$$


where

$$
\begin{aligned}
\mathscr{M}^{(k)} & =D_{\theta} \mathbf{g}^{(k)}\left(\theta_{0}\right), \\
\mathscr{L}^{(k)} & =\mathscr{P}\left[\mathscr{H}^{(1)} \Phi^{\left(k^{\prime}\right)}\left(\theta_{0}\right)+\ldots+\mathscr{H}^{\left(k^{\prime}+1\right)} \Phi^{(0)}\left(\theta_{0}\right)\right],
\end{aligned}
$$

and $k^{\prime}=(k-1) / 2$. For more details, we refer the interested reader to Pelinovsky et al. (2008). A prototypical example of a configuration (the so-called discrete diamond) that the above theory predicts to exist and be dynamically robust near the AC limit in 3D is illustrated in the bottom right panel of Fig. 1. Other such configurations can be found e.g. in Pelinovsky et al. (2008), as well as in Kevrekidis et al. (2004b) and Carretero-González et al. (2005).

\subsection{Future Perspectives}

Although, based on these recent results, much is presently understood about the statics and stability of DNLS type systems, a number of themes still remain relatively unexplored. We now present a few of the themes that we believe hold significant interest for future studies.

3.2.1 A general theory and applications of long-range interactions. While the setting of local (i.e., nearest-neighbor) interactions has been substantially explored in the DNLS case, this can far less be argued to be the case for the setting of non-nearest neighbor effects. Some early investigations of one dimensional settings identified the role of non-nearest neighbor interactions in creating bistability of the fundamental soliton solutions and leading to interesting possibilities for switching among stable branches (upon suitable perturbations) as in Johansson et al. (1998) and Christiansen et al. (1998). However, a more systematic investigation of existence and stability issues, especially for more complicated solutions was not offered.

More recently, a number of physically-minded works have argued the relevance of the inclusion of such non-nearest neighbor interactions in a variety of settings, involving predominantly optical waveguide arrays. For instance, the work of Efremidis \& Christodoulides (2002) argued that a zigzag waveguide array could be considered as a quasi-one-dimensional chain in which the relative strength of nearest-neighbor and next-nearest-neighbor interactions can be tuned on the basis of the relevant geometry. Corresponding ideas for involving non-nearest-neighbor interactions have also been generalized to two-dimensional settings. Although limited to the linear propagation at least in the realm of Szameit et al. (2008), even in that setting, there is interesting phenomenology arising from the competition of the different types of interactions, leading potentially to diffraction-free propagation for suitable wavenumbers in the center of the Brillouin zone. On the nonlinear side, the work of Kevrekidis (2009b) indicates that both the existence problem and the stability may be crucially modified with respect to the standard DNLS case by the presence of nonlocality. A typical example of the former involves the emergence of $1 \mathrm{D}$ solutions that have arbitrary (i.e., different than $0, \pi$ ) phases. A typical example of the latter involves the stabilization of unstable structures [such as a prototypical class of $S=2$ vortices in a square lattice of Pelinovsky et al. (2005b)]. It should also be noted that another area that may enhance the relevance of long-range interaction considerations is that of dipolar Bose-Einstein condensates (such as ${ }^{52} \mathrm{Cr}$ ) in the presence of optical lattices in atomic physics; see e.g. the recent review of Lahaye et al. (2009).

These directions and findings seem to warrant a more systematic investigation of the effects of nonlocality, ideally as a general function of properties (e.g. the "interaction range") of the kernel. Such 
a general setting could be:

$$
i \dot{u}_{n}=-\varepsilon \sum_{m=1}^{N} a_{n m} u_{m}-\left|u_{n}\right|^{2} u_{n}
$$

for different types of interaction kernels $a_{n m}$ (although the nonlocality could also in principle, or additionally be imposed on the nonlinear term). It would appear to be timely and relevant to explore how existence, stability and dynamics of solitary waves are progressively modified, as we depart from the well-understood local limit.

3.2.2 Intersite Lattices, Discretizations, Symmetries and Traveling Waves. One of the directions that also hold promise in the setting of DNLS models is that of different types of discretizations and their properties in comparison to the more fundamentally well-understood model with the centered-difference Laplacian and the onsite cubic nonlinearity. As a motivating example in this direction, we briefly discuss the recent report of Kevrekidis et al. (2009c) which examined the two-dimensional Ablowitz-Ladik model of the form:

$$
\begin{aligned}
i \dot{u}_{n, m}= & -\varepsilon\left(u_{n+1, m}+u_{n-1, m}+u_{n, m+1}+u_{n, m-1}-4 u_{n, m}\right) \\
& +\frac{\sigma}{4}|u|^{2}\left(u_{n+1, m}+u_{n-1, m}+u_{n, m+1}+u_{n, m-1}\right)
\end{aligned}
$$

The fundamental difference of this, so-called AL-NLS, model from the standard DNLS is that in addition to the centered-difference approximation of the Laplacian, a nearest-neighbor average is used to discretize the cubic nonlinearity of the continuum limit $\sigma|u|^{2} u$ (instead of a local term $\sigma\left|u_{n, m}\right|^{2} u_{n, m}$ in the DNLS). In the one-dimensional setting, such a centered-difference discretization of the nonlinearity is completely integrable, as shown by Ablowitz \& Ladik (1976), giving rise to exact hyperbolic-secant solitonic solutions that can travel at arbitrary speeds. This well-known (since the 1970s) fact already showcases the special properties that can emerge upon different types of discretization.

However, the recent work of Kevrekidis et al. (2009c) illustrated that surprising features may arise because of such discretizations in higher-dimensional settings as well. In particular, in the 2D case, it was found that the DNLS solitons become unstable at some critical threshold (e.g. of the coupling strength $\varepsilon$ ) as the continuum limit is approached (e.g. by increasing $\varepsilon$ ). The solitons then remain unstable all the way to the continuum limit (of $\varepsilon \rightarrow \infty)$ with the relevant instability eigenvalue approaching the origin of the spectral plane. This is because the $2 \mathrm{D}$ continuum NLS model is critical i.e., it is marginally unstable with respect to collapse. In the critical case, the continuum model is invariant under rescaling, see e.g. the relevant analysis of Sulem \& Sulem (1999). On the other hand, the AL-NLS approaches this continuum limit in a completely different way; in particular, while the AL-NLS two-dimensional solitons become unstable within a narrow range of parameters (such as $\varepsilon$ ), they subsequently become restabilized and remain stable as the continuum limit is approached. This suggests the remarkable fact that while the two models (DNLS and AL-NLS) are identical at the limit, infinitesimally close to the limit, their dynamics is substantially different. While the DNLS solitons are exponentially (yet weakly) unstable, the AL-NLS ones are dynamically stable.

Further consideration of this feature indicates that it is a particular trait of critical settings, which are at the very special separatrix between subcritical settings where the solitary waves are dynamically stable and supercritical ones, where the waves are exponentially unstable. In this critical case, the linear spectrum possesses an additional zero eigenvalue pair (associated with the pseudo-conformal invariance), which permits the reshaping of the solution under the action of the group of rescalings, and hence 
paves the way for the emergence of self-similar collapse. Discreteness can then shift this pair along the imaginary axis or along the real axis. The AL-NLS discretization turns out to be a prototypical example whereby the eigenvalue pair formerly associated with the pseudo-conformal invariance is perturbed in a stable way (moves along the imaginary axis of the spectral plane), upon discretization and hence this model allows infinitesimally small spacings to give rise to collapse-free dynamics.

The above discussion of issues pertaining to symmetry raises the more general question of devising different types of discretizations and examining their symmetry properties and the connection of these to the dynamical features of solitary waves. Assuming that discretizations of the continuum NLS model will respect the phase/gauge-invariance of that model, another key symmetry whose impact on discretizations has been examined fairly extensively recently is the invariance with respect to translations. Motivated by the early work of Speight \& Ward (1994), Speight (1999) and that of Kevrekidis (2003), there has been a considerable volume of literature developed recently on the subject of suitable discretizations of Klein-Gordon, as well as of NLS models which preserve (for their stationary solutions) the property of translational invariance; see e.g., Pelinovsky (2006) and references therein. It is well-known that such an invariance is absent in the standard DNLS model which admits single-humped discrete solitary waves which can only be centered on a site or half-way through between two sites (but not other such types of waveforms, contrary to what is the case with the translationally invariant models where the solitary waves can be centered anywhere). In this context, and for the NLS-model discretizations the work of Pelinovsky (2006) unified some of the earlier findings and methods, illustrating that the most general translationally invariant discretization of the cubic model is of the form:

$$
\begin{aligned}
i \dot{u}_{n}= & -\varepsilon\left(u_{n+1}+u_{n-1}-2 u_{n}\right)-(1-\chi-4 \xi-2 \eta)\left|u_{n}\right|^{2}\left(u_{n+1}+u_{n-1}\right)-\chi u_{n}^{2}\left(u_{n+1}^{\star}+u_{n-1}^{\star}\right) \\
& -\xi\left[\left(2\left|u_{n}\right|^{2}+\left|u_{n+1}\right|^{2}+\left|u_{n-1}\right|^{2}\right) u_{n}+\left(u_{n+1}^{\star} u_{n-1}+u_{n+1} u_{n-1}^{\star}\right) u_{n}+\left(u_{n+1}^{2}+u_{n-1}^{2}\right) u_{n}^{\star}\right] \\
& -\eta\left(\left|u_{n+1}\right|^{2}+\left|u_{n-1}\right|^{2}\right)\left(u_{n+1}+u_{n-1}\right)-v\left[u_{n+1}^{2} u_{n-1}^{\star}+u_{n-1}^{2} u_{n+1}^{\star}-\left|u_{n+1}\right|^{2} u_{n-1}-\left|u_{n-1}\right|^{2} u_{n+1}\right],
\end{aligned}
$$

where $(\chi, \xi, \eta, v)$ are real-valued parameters. When all four parameters vanish, one recovers the ALNLS limit.

This discussion, in turn, leads to the interesting additional question of the possibility of identifying traveling wave solutions in DNLS (and related) lattices. This question was considerably controversial during the previous decade with many contradictory results and conjectures; see e.g. the discussion in Melvin et al. (2006) and references therein. Starting from the work of Gómez-Gardeñes et al. (2004), which illustrated numerically that in DNLS such traveling waves cannot be localized, this question was subsequently answered both in the DNLS setting and in that of more complex models such as the saturable nonlinear proposed in Hadzievski et al. (2004) in the form:

$$
i \dot{u}_{n}=-\varepsilon \Delta_{2} u_{n}+\frac{1}{1+\left|u_{n}\right|^{2}} u_{n} .
$$

What the direct numerical simulations of Hadzievski et al. (2004) initially suggested and which was corroborated numerically in the work of Melvin et al. (2006), Melvin et al. (2008) and quasi-analytically through the asymptotic expansions of Oxtoby \& Barashenkov (2007) was that indeed in DNLS, the resonance of such traveling wave ("embedded soliton") type solutions with the (modified, for traveling solutions) continuous spectrum gave rise to "nanoptera" i.e., solutions with a non-vanishing tail. However, in different types of nonlinearities such as the saturable or cubic-quintic ones, these works showed that it is possible to make the prefactor of such a resonance (the so-called Stokes constant) vanish, thereby producing non-generic, yet exact, exponentially localized traveling solutions in these models. 
The above findings, in turn, beg the somewhat open-ended question: is there some simple diagnostic which may indicate the existence of such traveling solutions in a model? The works of Hadzievski et al. (2004) and Melvin et al. (2006) [see also the studies of Carretero-González et al. (2006) and Chong et al. (2009) for corresponding discussions of the cubic-quintic discrete model] hinted at the use of the socalled Peierls-Nabarro barrier, i.e., the energy difference between on-site and inter-site centered solutions. In particular, they argued that vanishings of this barrier should be physically expected to be connected with the possibility of such traveling solutions. However, this connection has not been made rigorous, as of yet. More generally, exploring systematically the connection of Peierls-Nabarro barriers, the existence of traveling solutions, the calculation of the Stokes constant, and the potential for underlying symmetries (including a possible semi-discrete type of Galilean invariance) could be extremely interesting topics for further exploration over the next decade. Understanding such properties either on a model-specific basis, or, ideally, based on more general/fundamental principles is an important open direction for these nonlinear dynamical lattices. At this point, we should also mention in passing the very interesting recent work of Barashenkov \& van Heerden (2008). There, the examination of exceptional discretizations [bearing an effective translational invariance through the map type approach of Pelinovsky (2006) and Barashenkov et al. (2005)] led to some ingenious suggestions on how to discretize so as to preserve genuinely traveling localized excitations i.e., kinks in the discrete sine-Gordon and discrete $\phi^{4}$ settings. It would be extremely worthwhile for such methodologies to be brought to bear also in DNLS and related settings.

3.2.3 Statistical Mechanics of the DNLS and Approach to Equilibrium. One of the questions that has received a somewhat limited amount of attention is that of the statistical mechanics of the DNLS model and how a given initial condition approaches its asymptotic (equilibrium or near-equilibrium) dynamics. One of the early studies of such questions for the DNLS lattice appeared in Rasmussen et al. (2000). There, a regime in phase space was identified wherein regular statistical mechanics considerations apply, and hence, thermalization was observed numerically and explored analytically using regular, grandcanonical, Gibbsian equilibrium measures. However, the nonlinear dynamics of the problem renders permissible the realization of regimes of phase space which would formally correspond to "negative temperatures" in the sense of statistical mechanics. The novel feature of these states was found to be that the energy spontaneously localizes in certain lattice sites forming breather-like excitations (as observed numerically and experimentally). Returning to statistical mechanics, such realizations are not possible [since the Hamiltonian is unbounded, as is seen by a simple scaling argument similar to the continuum case studied in Lebowitz et al. (1988)] unless the grand-canonical Gibbsian measure is refined to correct for the unboundedness. This correction was argued in Rasmussen et al. (2000) to produce a discontinuity in the partition function signaling a phase transition which was identified numerically by the appearance of discrete breathers.

More recently, the statistical understanding of the formation of localized states and of the asymptotic dynamics of the DNLS equation has been examined in the works of Rumpf (2004) and Johansson \& Rasmussen (2004). Using small-amplitude initial conditions, Rumpf (2004) argued that the phase space of the system can be divided roughly into two weakly interacting domains, one corresponding to the lowamplitude fluctuations (linear or phonon modes), while the other consists of the large-amplitude, localized mode nonlinear excitations. Then, based on a simple partition of the energy $H=H_{-}+H_{+}$and of the norm $P=P_{-}+P_{+}$, into these two broadly (and also somewhat loosely) defined fractions, one smaller than a critical threshold (denoted by "-") and one larger than a critical threshold (denoted by "+"), it is possible to compute thermodynamic quantities such as the entropy in this localization regime. In particular, one of the key results of the work of Rumpf (2004) is that, for a partition of $K$ sites with 
large amplitude excitations and $N-K$ sites with small amplitude ones, an expression is derived for the total entropy (upon computing $S_{-}, S_{+}$and a permutation entropy due to the different potential location of the $K$ and $N-K$ sites). This expression reads:

$$
S=N \ln \Omega+\frac{P_{+}^{2}}{2 E_{+}} \ln \Gamma
$$

where $\Omega=\left(4 P_{-}^{2}-E_{-}^{2}\right) /\left(4 A_{-}(N-K)\right)$ and $\Gamma=2 P_{-} N / P_{+} E_{-}$, while $K=P_{+}^{2} /\left(2 E_{+}\right)$. While some somewhat artificial assumptions are needed to arrive at the result of Eq. (3.25) (such as the existence of a cutoff amplitude radius $R$ in phase space), nevertheless, the result provides a transparent physical understanding of the localization process. The contributions to the entropy stem from the fluctuations [first term in Eq. [3.25)] and from the high amplitude peaks (second term in the equation). However, typically the contribution of the latter in the entropy is negligible, while they can absorb high amounts of energy. The underlying premise is that the system seeks to maximize its entropy by allocating the ideal amount of energy $H_{-}$to the fluctuations. Starting from an initial energy $H_{-}$, this energy is decreased in favor of localized peaks (which contribute very little to the entropy). The entropy would then be maximized if eventually a single peak was formed, absorbing a very large fraction of the energy while consuming very few particles. Nevertheless, practically, this regime is not reached "experimentally" (i.e., in the simulations). This is because of the inherent discreteness of the system which leads to a pinning effect of large amplitude excitations which cannot move (and, hence, cannot eventually merge into a single one) within the lattice. Secondly, the growth of the individual peaks, as argued in Rumpf (2004), stops when the entropy gain due to energy transfer to the peaks is balanced by the entropy loss due to transfer of power. While placing the considerations of Rumpf (2004) in a more rigorous setting is a task that remains open for future considerations, this conceptual framework offers considerable potential for understanding the (in this case argued to be infinite, rather than negative, temperature) thermal equilibrium state of coexisting large-amplitude localized excitations and small-amplitude background fluctuations.

On the other hand, the work of Johansson \& Rasmussen (2004) extended the considerations of the earlier work of Rasmussen et al. (2000) to the generalized DNLS model of the form:

$$
i \dot{u}_{n}+\left(u_{n+1}+u_{n-1}\right)+\left|u_{n}\right|^{2 \sigma} u_{n}=0,
$$

with $\sigma$ being a free parameter within the nonlinearity exponent. Furthermore, in the work of Johansson \& Rasmussen (2004), the connection of these DNLS considerations with the generally more complicated KleinGordon (KG) models was discussed. Much of the above mentioned phenomenology, as argued in Rumpf (2004), is critically particular to NLS type models, due to the presence of the second conserved quantity, namely of the $l^{2}$ norm; this feature is absent in the KG lattices, where typically only the Hamiltonian is conserved. Johansson \& Rasmussen (2004) formalize the connection of DNLS with the KG lattices, by using the approximation of the latter via the former through a Fourier expansion whose coefficients satisfy the DNLS up to controllable corrections. Within this approximation, they connect the conserved quantity of the KG model to the ones of the DNLS model approximately reconstructing the relevant transition (to formation of localized states) criterion discussed above. However, in the KG setting this only provides a guideline for the discrete breather formation process, as the conservation of the norm is no longer a true but merely an approximate conservation law. This is observed in the dynamical simulations of Johansson \& Rasmussen (2004), where although as the amplitude remains small throughout the lattice the process is well described by the DNLS formulation, when the discrete breathers of the KG problem grow, they violate the validity of the DNLS approximation and of the norm conservation; thus, a description of the asymptotic state and of the thermodynamics of such lattices requires further 
elucidation that necessitates a different approach. This is another interesting and important problem for future studies.

It is also worth pointing out that many of the considerations of works such as that of Rasmussen et al. (2000) or of Johansson \& Rasmussen (2004) are intrinsically one-dimensional in nature and can not be straightforwardly generalized (per the nature of the transfer integral technique used) to higher dimensions. Hence, the issue of the asymptotic dynamics is perhaps more pressing in such higher-dimensional settings, especially given their connections to collapse (sufficiently close to the continuum limit). But even in one-dimension, numerous questions remain. One such interesting question was raised by the recent work of Kevrekidis et al. (2008b). This work offers an understanding of the fundamental threshold (for initial data supported on a single site) between discrete dispersion for sub-critical initial amplitude and nonlinearity-induced localization, for sufficiently high initial amplitude. However, it also poses the question: once we know localization will ensue, which "member" of the mono-parametric family of localized solutions will the dynamics "select" for the asymptotic relaxation state? Numerical experiments illustrate that the initial condition will shed both some mass and some energy, eventually converging to one particular member of that family of stationary solutions. But what is missing is the guiding principle of such a selection. Interestingly, while the single-site supported initial data can give localization in the DNLS model (when it possesses a sufficiently large amplitude), in the AL-NLS model, such data always leads to dispersion. In the latter case, the machinery of the theory of integrability can be brought to bear to appreciate the effect of different types of compactly supported initial conditions as shown in Espinola-Rocha \& Kevrekidis (2009).

3.2.4 Interplay of Nonlinearity and Disorder and its Implications on Anderson Localization. One of the particularly interesting recent developments on the front of DNLS equations has been the examination of the interplay of nonlinearity with disorder, in an effort to explore how the presence of the former affects the fundamental phenomenon of Anderson localization due to the latter as first illustrated in Anderson (1958). This topic has spurred a significant controversy within the physics and mathematics communities, given its fundamental nature and the somewhat conflicting results reported recently. In particular, a number of recent experimental studies in optical [see the works of Schwartz et al. (2007) and Lahini et al. (2008)] and atomic [see e.g. the work of Sanchez-Palencia et al. (2007)] physics settings have reported the observation of the linearly induced exponential localization proposed by Anderson for a disordered linear lattice. Nevertheless, in a series of recent computational publications in the physics literature by at least two separate groups (whose theoretical arguments produce different results) in Kopidakis et al. (2008), Flach et al. (2009), and Pikovsky \& Shepelyansky (2008), it has been argued that nonlinearity essentially destroys Anderson localization by producing a subdiffusive scaling of a quantity such as the second moment $M_{2}=\sum\left(n-n_{0}\right)^{2}\left|u_{n}(t)\right|^{2}$ (where $\left|u_{n}(t)\right|^{2}$ plays the role of the wavefunction density in the Schrödinger problems considered therein). This subdiffusive scaling implies that progressively more distant lattice sites are occupied (although slowly) and the process is not stopped by the trapping anticipated on the basis of the Anderson mechanism. More specifically, it has recently been argued in Flach et al. (2009) that there exist three regimes within the system's spectral dynamics. For weak nonlinearity (smaller than necessary to induce transitions between linear modes), the system is in a transient Anderson localization regime, but eventually it detraps from it and grows according to the subdiffusive scaling $M_{2}(t) \sim t^{\alpha}$, with $\alpha<1$. For intermediate nonlinearities, the subdiffusion of $M_{2}$ is initiated immediately, while large nonlinearity leads to localization of a fraction of the initial wavepacket, while the "radiative" remainder is also subject to this subdiffusive expansion. In the more recent work of Skokos et al. (2009), the relevant considerations were extended to more complex initial data (not only of single site, but also of single mode or finite size), and also a similar phenomenology 
was proposed in Krimer et al. (2009) to arise in the setting of DNLS equations with linear (so-called Stark ladder) potentials. In the latter, a discrete, equidistant spectrum is also known to arise (but now due to the linear potential), hence the same subdiffusion type dynamics is expected to ensue in the presence of nonlinearity. This controversy is extremely interesting, since it highlights the potential "fragility" of the Anderson localization regime, indicating that even very weak nonlinearity is sufficient to eliminate the relevant phenomenology.

However, all the above considerations, at present, stay within the realm of (admittedly, very strongly suggestive) numerical experiments. Neither real experiments (e.g., with optical waveguides or BECs in optical lattices) or rigorous mathematical arguments have been provided to support this subdiffusional behavior. It would be particularly interesting to try to examine such issues from a rigorous point of view, also considering how the relevant subdiffusive scalings may depend on the form (and distribution) of the random perturbations. Would similar phenomena also arise in the presence of nonlinear random perturbations? Could the integrability of the underlying nonlinear model crucially alter the dynamics (e.g., what would happen to the above results if the DNLS was changed to the AL-NLS model)? These are only some of the numerous questions that this novel direction raises.

\section{Klein Gordon Models in Mechanical and Electrical Systems}

\subsection{Recent Developments}

Interestingly, in recent years, there has been a parallel stream of developments examining the dynamics of the localized modes (discrete breathers - DBs_-) which are in some sense the analogs of the standing waves of the DNLS equation considered in the previous section. Some of the fundamental tools that enabled the consideration of the existence and stability theory of the discrete breathers and multibreathers were seeded in the original work of Ahn et al. (2001) and subsequently were generalized e.g. in that of MacKay \& Sepulchre (2002). Similar results about the stability of such states had been obtained earlier in Archilla et al. (2003), using the notion of the so-called Aubry band theory developed in Aubry (1997), however the results of the two methods can be shown to be equivalent. In the presentation that follows, we will be using the formulation of MacKay and collaborators, as was adapted to the KG setting by Koukouloyannis \& Kevrekidis (2009). This has the benefit that not only does it allow to quantify the existence conditions of the relevant structures, but it also offers tantalizing connections of the KG setting with the previously explored DNLS one. It should also be noted that in this setting, as well, the relevant results have not been restrained to one-dimensional or just square lattices, but have, in fact, been generalized to non-square lattices, such as hexagonal and honeycomb ones in the works of Koukouloyannis \& MacKay (2005), Koukouloyannis \& Kourakis (2009) and Koukouloyannis et al. (2009).

A short outline of the relevant methodology is as follows. Assume that the system Hamiltonian is of the form

$$
H=H_{0}+\varepsilon H_{1}=\sum_{i=-\infty}^{\infty}\left(\frac{1}{2} p_{i}^{2}+V\left(x_{i}\right)\right)+\frac{\varepsilon}{2} \sum_{i=-\infty}^{\infty}\left(x_{i+1}-x_{i}\right)^{2}
$$

where the part of $H_{0}$ is associated with an unperturbed single oscillator, and $H_{1}$ denotes the perturbing part due to the coupling with the neighboring oscillators. Considering a number of central excited oscillators, one can write their solution $x(t)$ into the action-angle form $x(t)=\sum_{n=0}^{\infty} A_{n}(J) \cos (n w)$, where $J, w$ are the action-angle variables. Then, over the $n$ excited oscillators, one can define the effective 
Hamiltonian

$$
H^{\text {eff }}=H_{0}\left(I_{i}\right)+\varepsilon\left\langle H_{1}\right\rangle\left(\phi_{i}, I_{i}\right) \quad i=1 \ldots n,
$$

over the canonical variables:

$$
\begin{array}{ll}
\vartheta=w_{0} & \mathscr{A}=J_{0}+\ldots+J_{n} \\
\phi_{1}=w_{1}-w_{0} & I_{1}=J_{1}+\ldots+J_{n} \\
\phi_{2}=w_{2}-w_{1} & I_{2}=J_{2}+\ldots+J_{n} \\
\vdots & \vdots \\
\phi_{n}=w_{n}-w_{n-1} & I_{n}=J_{n}
\end{array}
$$

and with

$$
\left\langle H_{1}\right\rangle=\frac{1}{T} \oint H_{1} \mathrm{~d} t
$$

where the integration is along the unperturbed periodic orbit.

One of the key results of the theory of MacKay and collaborators is that the critical points of this effective Hamiltonian yield the DB solutions of the model. In the KG setting discussed above, Koukouloyannis \& Kevrekidis (2009) derived the effective Hamiltonian in the form:

$$
\left\langle H_{1}\right\rangle=-\frac{1}{2} \sum_{m=1}^{\infty} \sum_{i=1}^{n} A_{m}^{2} \cos \left(m \phi_{i}\right)
$$

and hence obtained the persistence conditions through $\frac{\partial\left\langle H_{1}\right\rangle}{\partial \phi_{i}}=0$. Remarkably, in this setting, similarly to DNLS, it can again be proved that the only available multi-pulse (i.e., multi-breather) solutions are the ones with relative phase among the excited sites of 0 or $\pi$.

Subsequently, one can examine the stability of these DB solutions, which is controlled by the Hessian of the effective Hamiltonian presented above. In particular, $\mathbf{E}=\mathbf{J} D^{2} H^{\text {eff }}$ where the symplectic matrix reads $\mathbf{J}=\left(\begin{array}{cc}\mathbf{O} & -\mathbf{I} \\ \mathbf{I} & \mathbf{O}\end{array}\right)$. Once again, an explicit calculation of the relevant stability matrix yields:

$$
\mathbf{E}=\left(\begin{array}{c|c|c}
\mathbf{A} & \mathbf{B} \\
\hline \mathbf{C} & \mathbf{D}
\end{array}\right)=\left(\begin{array}{c|c}
\varepsilon \mathbf{A}_{\mathbf{1}} & \varepsilon \mathbf{B}_{\mathbf{1}} \\
\hline \mathbf{C}_{\mathbf{0}}+\varepsilon \mathbf{C}_{\mathbf{1}} & \varepsilon \mathbf{D}_{\mathbf{1}}
\end{array}\right)=\left(\begin{array}{cc}
-\varepsilon \frac{\partial^{2}\left\langle H_{1}\right\rangle}{\partial \phi_{i} \partial I_{j}} & -\varepsilon \frac{\partial^{2}\left\langle H_{1}\right\rangle}{\partial \phi_{i} \partial \phi_{j}} \\
\hline \frac{\partial^{2} H_{0}}{\partial I_{i} I_{j}}+\varepsilon \frac{\partial^{2}\left\langle H_{1}\right\rangle}{\partial I_{i} \partial I_{j}} & \varepsilon \frac{\partial^{2}\left\langle H_{1}\right\rangle}{\partial \phi_{j} \partial I_{i}}
\end{array}\right) .
$$

For the special case of relative phases $\phi_{i}=0$, or $\phi_{i}=\pi$, the matrix simplifies considerably acquiring the form:

$$
\mathbf{E}=\left(\begin{array}{ll}
\mathbf{O} & \mathbf{B} \\
\mathbf{C} & \mathbf{O}
\end{array}\right)=\left(\begin{array}{cc}
\mathbf{O} & \varepsilon \mathbf{B}_{1} \\
\mathbf{C}_{\mathbf{0}}+\varepsilon \mathbf{C}_{\mathbf{1}} & \mathbf{O}
\end{array}\right)
$$

which, subsequently, if we consider only the dominant eigenvalue contributions (where $\lambda^{2}$ is of $\mathscr{O}(\varepsilon)$ ), yields the relevant squared eigenvalue matrix in the form:

$$
\mathbf{B}_{\mathbf{1}} \cdot \mathbf{C}_{\mathbf{0}}=-\frac{\partial \omega}{\partial J} \mathbf{Z}=-\frac{\partial \omega}{\partial J}\left(\begin{array}{ccccc}
2 f_{1} & -f_{1} & 0 & & \\
-f_{2} & 2 f_{2} & -f_{2} & 0 & \\
& \ddots & \ddots & \ddots & \\
& 0 & -f_{n-1} & 2 f_{n-1} & -f_{n-1} \\
& & 0 & -f_{n} & 2 f_{n}
\end{array}\right)
$$



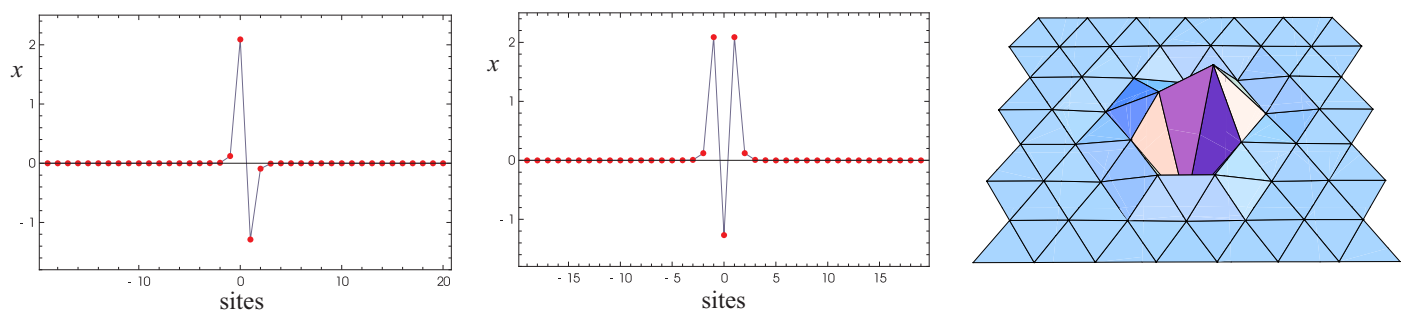

FIG. 2. (Color online) A sampler of breather solutions of the discrete nonlinear Klein-Gordon equation in one and two spatial lattice dimensions with a (soft) potential of the type used in the work of Koukoulovannis \& Kevrekidis (2009). The left panel shows an out-of-phase two-site mode, while the middle an out-of-phase three-site mode. The right panel shows a two-dimensional hexagonal lattice generalization of these modes in the form of a discrete vortex breather $(2 \pi / 3$ phase difference between the three adjacent excited oscillators), per the work of Koukouloyannis \& Kourakis (2009). All three structures are stable close to the anti-continuum limit.

In this expression $\omega=\partial H_{0} / \partial J$ denotes the frequency, while $f_{i}=f\left(\phi_{i}\right)=(1 / 2) \sum_{n=1}^{\infty} n^{2} A_{n}^{2} \cos \left(n \phi_{i}\right)$. This leads to the characteristic exponents (i.e., effective eigenvalues) of the DB in the form:

$$
\sigma_{ \pm i}= \pm \sqrt{\varepsilon \chi_{1 i}}+\mathscr{O}\left(\varepsilon^{3 / 2}\right) \quad i=1 \ldots n
$$

where $\chi_{1 i}=-\frac{\partial \omega}{\partial J} z_{i}$ and $z_{i}$ are the eigenvalues of the matrix $\mathbf{Z}$ defined above. Remarkably, the count of unstable eigenvalues comes out to be identical to the DNLS case, for the focusing or soft nonlinearities which satisfy $\varepsilon \frac{\partial \omega}{\partial J}<0$. That is, the number of unstable eigendirections will be precisely equal to the number of nearest neighbors which are in phase between them. Lastly, if the sign of the above product $\varepsilon \frac{\partial \omega}{\partial J}$ changes, then the conclusion indicates that the number of unstable eigendirections is precisely equal to the number of out-of-phase nearest neighbors. Some typical examples of the relevant configurations in one- and two-dimensional settings are shown in Fig. 2

\subsection{Future Perspectives}

While the general Hamiltonian framework of discrete breathers in Klein-Gordon lattices is gradually being explored at a fundamental level regarding the existence and stability properties that it entails for its coherent structures, numerous new applications are emerging in this field which require considerable additional modeling efforts and which bear significant differences from this general framework.

One such example is the recent work of Cuevas et al. (2009), where the dynamics of discrete breathers were examined in arrays of coupled pendula, in the more realistic experimental setting of forcing and damping. In the Hamiltonian setting the relevant model for this system is simply the discrete sine-Gordon equation, where the linear coupling stems from the torsional springs, and the sinusoidal nonlinearity from the gravitationally induced torsion. However, the actual forced-damped array is more accurately described [as argued in Cuevas et al. (2009)] by:

$$
\ddot{u}_{n}+\omega_{0}^{2} \sin \left(u_{n}\right)-\frac{\beta}{I} \Delta_{2} u_{n}=-\frac{\gamma}{I} \dot{u}_{n}+\frac{\gamma_{2}}{I} \Delta_{2} \dot{u}_{n}-F \omega_{d}^{2} \cos \left(\omega_{d} t\right) \cos \left(u_{n}\right),
$$

where, in addition to the intrinsic frequency $\omega_{0}$ of each pendulum and their torsional coupling of strength $\beta$, terms for the damping of each pendulum (characterized by $\gamma$ ), the viscous damping due to their 
coupling (characterized by $\gamma_{2}$ ) and the strength $F$ of their parametric drive (characterized also by the frequency $\omega_{d}$ ) have been included; $I$ denotes the pendulum's moment of inertia. In this context, a number of interesting features have been highlighted in the work of Cuevas et al. (2009), including the apparent exchange of stability between the onsite (i.e., site-centered) breather, and the inter-site (i.e., bond-centered) form. Both of these waveforms were experimentally observed, each in their range of stability, illustrating that there is a separatrix between the respective ranges. The existence of such a separatrix connects partially also to the discussion of the previous section about the Peierls-Nabarro barriers, and their disappearance, as well as of the latter leading to mobility of the localized modes. The mobility of discrete breathers was, in fact, also observed in Cuevas et al. (2009) both experimentally and numerically.

Nevertheless, there are still numerous topics within this theme that remain partially unexplored in this more elaborate, yet more realistic class of models. On the one hand, it would be useful to explore some of the instabilities giving rise to coherent structures therein, such as the modulational instability. Experiments already show the presence of such phenomena as analyzed by English (2009), but it would be useful if a quantitative description thereof could be provided. Another interesting direction worth expanding towards is that of multi-pulses. From the general theory of the previous subsection, an understanding is emerging about which multi-pulse solutions (and under what phase differences) should be expected to be stable/dynamically robust. Developing a similar framework of understanding for the forced-damped setting initially from a computational perspective (and possibly also analytically at a later stage) would certainly be desirable.

A similar driven damped setting has recently been explored in the context of driven and damped electrical lattices in the work of Sato et al. (2007) and of English et al. (2008). There the experimental system is a bi-inductive discrete electrical transmission line, which is nonlinear due to the nonlinear dependence of the capacitance of the diode on the voltage and which leads to the following dynamical model:

$$
\begin{aligned}
\ddot{u}_{n}+\left(\omega_{0}^{2} u_{n}-\frac{\omega_{0}^{2}}{2 Q_{0}} u_{n}^{2}+\frac{\omega_{0}^{2}}{3 Q_{0}^{2}} u_{n}^{3}\right)+\omega_{1}^{2} \Delta_{2} u_{n}= & -\frac{\omega_{1}^{2}}{2 Q_{0}} \Delta_{2} u_{n}^{2}+\frac{\omega_{1}^{2}}{3 Q_{0}^{2}} \Delta_{2} u_{n}^{3}+\frac{1}{R} \frac{d V_{d}}{d t} \\
& +\frac{1}{R C_{0}}\left[1-\left(\frac{u_{n}}{Q_{0}}\right)+\left(\frac{u_{n}^{2}}{Q_{0}^{2}}\right)\right] .
\end{aligned}
$$

Various electrical constants are denoted by their respective symbols ( $R$ for resistance, $C_{0}$ for capacitance and $Q_{0}$ for charge), while the external driver is given by $V_{d}$. Some of the very interesting features observed in this system include how its discrete breathers could interact with impurities (being e.g. seeded by or attracted to a capacitor, while they could be destroyed or repelled by inductors and/or resistors). Also, it was found that depending on the driver amplitude and especially the driver frequency, the system would spontaneously lock into 1- or 2- or multi-breather states within a finite size (e.g. with 24 nodes) experimental lattice. It would be extremely interesting to try to explore the existence and the stability of localized modes within such transmission line systems and to explore the origin of the observed pattern selection and dynamics. To the best of our knowledge, very little has been done theoretically in this direction for such more realistic systems.

Interestingly, electrical lattices constitute, arguably, the simplest realization of one of the focal points of interest in the last few years (which is likely to be one of the major areas of development for the next decade), namely of nonlinear metamaterials Kozyrev \& van der Weide (2008). Such media have simultaneously negative electric permittivity and magnetic permeability (so that their root square product, i.e., the speed of light propagation is real); however, because of these negative values, they feature a num- 
ber of unusual effects, including negative refraction, opposite signs of group and phase velocity (i.e., wavefronts and wavepackets move in opposite directions), inverse Doppler effect, backward Cerenkov radiation, etc. A nice introduction to this field can be found e.g. in the work of Marques et al. (2008) [see also for a review with a more nonlinear slant the presentation of Lichtnitser \& Shalaev (2008)]. One of the simplest (if not the simplest) lattice that produces such backward waves can be generated by feeding a ladder network with alternating shunt-connected inductors and series capacitors [as shown e.g. in Fig. 3.1 of Marques et al. (2008)]. However, recent work in the realm of nonlinear waves has mostly focused on the slightly more elaborate setting of magnetic metamaterials and, in particular, lattices of split-ring resonators. The resonators are electrically equivalent to an RLC oscillator, with self-inductance $L$, ohmic resistance $R$ and capacitance $C$ and become nonlinear because of the gaps that they possess which are filled with a nonlinear dielectric medium (whose permittivity depends on the intensity of the electric field $E$ ). The relevant mathematical description of the dynamics of the charge stored on the capacitor of the $n$-th split-ring resonator (SRR) of such a SRR lattice, coupled to the current circulating through it can be obtained as follows:

$$
\begin{aligned}
\frac{d Q_{n}}{d t} & =I_{n} \\
\frac{L d I_{n}}{d t} & =-I R_{n}+M\left(\frac{d I_{n+1}}{d t}+\frac{d I_{n-1}}{d t}\right)-f\left(Q_{n}\right)+\mathscr{E} .
\end{aligned}
$$

Here, $\mathscr{E}$ is the electromotive force (i.e., external drive) induced in each SRR due to the applied field, while $f\left(Q_{n}\right)$ bears the nonlinearity of the model since the field-dependent permittivity gives rise to a field dependent capacitance, which in turn leads to its nonlinear dependence on the charge. $f$ can be reasonably approximated by an odd power series in $Q_{n}$, as is illustrated e.g. in the work of Lazarides et al. (2006). This formulation of, once again, a damped and driven electrical lattice has been used in the work of Lazarides et al. (2006) to predict the existence of dissipative breathers (as well as Hamiltonian ones, in the absence of the driving and damping) under an AC (alternating current) electromotive force. This work initiated a path towards a more systematic exploration of such structures, which was continued in the work of Eleftheriou et al. (2009), examining such structures in both 1- and 2-dimensional settings, that of Maluckov et al. (2008) for different types of nonlinearities, of Lazarides et al. (2008) and Eleftheriou et al. (2009) for surface variants of these discrete breathers and of Molina et al. (2009) for binary metamaterials. More recently, it should be mentioned that further "frequency gap" regions in such metamaterials have been explored e.g. in the work of Tsitsas et al. (2010), where the signs of the magnetic permeability and dielectric permittivity are opposite; this regime has been predicted to also possess breathers due to its approximate description by the so-called short-pulse equation [see e.g., Schaeffer \& Wayne (2004)]. However, it should be mentioned that in most of these settings, the relevant models are derived, and subsequently are analyzed in a purely numerical form. Both a theoret$\mathrm{ical} / \mathrm{mathematical}$ analysis of the types of structures that are possible, and experimental realizations of the relevant settings are presently lagging behind and would be a natural direction for future work.

\section{FPU-type Models in Granular Crystals and Dusty Plasmas}

\subsection{Recent Developments \& Future Perspectives}

FPU models have a time-honored history of more than half a century, one that has been captured in both special volumes such as that of Campbell et al. (2005), as well as in recent books such as the one by Galavotti (2008). Despite the interesting recent developments in this area, including the $q$-breathers 
of Flach et al. (2005) (which may be closely related to the original FPU paradox), here we will not focus on this intensely-studied theme. Instead, we will present some particular recent directions within the same general class of models which feature predominantly intersite nonlinear interactions within a lattice setting.

The principal example of the above class that we will consider is that of the so-called "granular crystals", namely arrangements of densely packed spheres, which upon suitable excitations interact elastically through the so-called Hertzian contacts, and result in the propagation of compression waves (or, as we will see, the localization of suitable breather-like states) in these media. These chains have drawn considerable attention during the past few years. This broad interest has emerged, to a considerable extent, due to the wealth of available material types/sizes and the ability to tune their dynamic response to encompass linear, weakly nonlinear, and strongly nonlinear regimes as shown in the works of e.g. Nesterenko (2001), Sen et al. (2008), Daraio et al. (2006) and Coste et al. (1997). Such flexibility makes them perfect candidates for many engineering applications, including shock and energy absorbing layers proposed e.g. in the works of Daraio et al. (2006), Hong (2005), Fraternali et al. (2010) and Donev \& Sen (2006); actuating devices [see e.g. Khatri et al. (2008)]; and sound scramblers [see e.g. Daraio et al. (2005) and Nesterenko et al. (2005)].

As an aside, we should note here that in an area such as that of granular crystals, which from the perspective of mathematical results is still at an early stage (most of the focus has been on numerical investigations and experimental results), it is not easy to distinguish between recent developments and future perspectives. For this reason, (and differently than in the previous sections) we will present both of these in a unified format in what follows.

The model used to represent the simplest form of such granular systems consists of a one dimensional (1D) chain of spherical beads regulated by Hertzian contact interaction potentials, see e.g. Johnson (2004). For this class of models, a nonlinear wave theory, supporting nearly-compact solitary waves was derived for all structured homogeneous [as discussed e.g. in Nesterenko (2001)] and later also for heterogeneous periodic [as illustrated in Porter et al. (2008)] materials showing a highly nonlinear force $(F)$-displacement $(\delta)$ response dictated by the intrinsically nonlinear potential of interaction between its fundamental components. These general nonlinear spring-type lattice interactions read:

$$
F=-V^{\prime}\left(u_{i}-u_{i-1}\right)=A \delta^{p}, \quad \delta=\max \left(0, u_{i-1}-u_{i}\right), \quad \ddot{u}_{i}=-V^{\prime}\left(u_{i}-u_{i-1}\right)+V^{\prime}\left(u_{i+1}-u_{i}\right)
$$

where $A$ depends on the material properties and $p$ is the nonlinear exponent of the contact interaction (with $p>1$ ); $p=3 / 2$ for spheres (Hertzian interactions). By granular matter, we mean an aggregate of particles in elastic contact with each other, preferably in linear or network shaped arrangements as discussed e.g. in Goddard (1990), Gilles \& Coste (2001), Goldenberg \& Goldhirsch (2005) and Hostler \& Brennen (2005). In addition to the nonlinear contact interaction, and related purely to the particle geometry, another unusual feature of the granular state is the zero tensile strength, which introduces an additional nonlinearity (asymmetric potential) to the overall response. In the absence of static precompression, these properties result in an essentially nonlinear force which leads to interesting implications on the dynamics and a vanishing sound speed $c_{0}=0$ (so-called "sonic vacuum"); see e.g. Vakakis et al. (2008). This makes the linear and weakly nonlinear continuum approaches based on the Korteweg-de Vries (KdV) equation invalid and places granular crystals in a strongly nonlinear regime of wave dynamics. This feature uncovers a significant potential for relevant applications. In particular, it supports a new type of nearly compact highly tunable solitary waves that have been experimentally and numerically observed for 1D Hertzian granular systems as summarized e.g. in the reviews of Nesterenko (2001) and Sen et al. (2008). While spherical bead crystals have been mostly studied, similar responses can be obtained from cylindrical and elliptical particles [see e.g. Daraio et al. (2009)], fibrous layers 
[see e.g. Lambert \& Tesar (1984)] and foams [see e.g. Sen et al. (2008)].

The prototypical setup of Hertzian contacts describing elastic interactions among grains of a unary (i.e., monoatomic) lattice in the absence of any precompression (i.e., in the so-called sonic-vacuum regime) can be written as:

$$
\ddot{u}_{n}=\left[u_{n-1}-u_{n}\right]_{+}^{p}-\left[u_{n}-u_{n+1}\right]_{+}^{p} .
$$

Here, the $[\cdot]_{+}$notation indicates that that the quantity in the bracket is only evaluated if positive, while it is set to 0 , if negative. Reformulating the problem based on the strain variant of the equation for $r_{n}=u_{n-1}-u_{n}$, we obtain

$$
\ddot{r}_{n}=\left[r_{n+1}\right]_{+}^{p}-2\left[r_{n}\right]_{+}^{p}+\left[r_{n-1}\right]_{+}^{p} .
$$

If we now seek traveling wave solutions, as suggested by the above mentioned numerical and experimental work (as well as the near-continuum asymptotics used) by numerous authors [see e.g. the presentations of Nesterenko (2001) and Sen et al. (2008)], then $r_{n}=\phi(x) \equiv \phi(n-c t)$. This leads to an advance-delay equation the solution of which, from a rigorous viewpoint, "remains an open question and apparently a delicate one" as indicated by English \& Pego (2005). This is because, although the latter problem can be reformulated as an iterative scheme of the form:

$$
\phi_{m}(x)=\frac{\left(\Lambda \cdot \phi_{m-1}^{p}\right)(x)}{\int_{-1}^{1}\left(\Lambda \cdot \phi_{m-1}^{p}\right)(x) d x}
$$

with $\Lambda(x)=[1-|x|]_{+}$, the proof of existence of a localized solution thereof for any $p>1$ is presently missing. Nevertheless, from a numerical perspective, the scheme of Eq. (5.4) has been shown to converge to the desired solutions in the works of English \& Pego (2005) and Ahnert \& Pikovsky (2009); for an example of the relevant traveling wave in the Hertzian case, see Fig. 3 It should also be mentioned that should such a proof be available, it would immediately imply that

$$
r(x+1) \leqslant \sup _{y \in[-1,1]} r^{p}(x+1-y) \int_{-1}^{1} \Lambda(x)=r^{p}(x),
$$

which, in turn, suggests the double exponential law $r(x+n) \leqslant r(x)^{p^{n}}$ for the decay of the solution tail. It is this extremely fast decay which leads the continuum analog of the solution to sustain compactly supported structures. However, it should be noted that this latter statement has not been rigorously proven either (to the best of our knowledge). In fact, there are multiple continuum models that have been proposed for the system dynamics. The most standard of them stems from the long wavelength approximation (LWA) applied to the displacement problem by Nesterenko (2001) [see also Coste et al. (1997)], while a more recent approach in the work of Ahnert \& Pikovsky (2009) suggests that the LWA be applied to the equation for the strains. The former approach leads to the partial differential equation (PDE), for the strain variables, of the form:

$$
r_{t t}=\left(r^{p}\right)_{x x}+\frac{\varepsilon^{2}}{12}\left(\left(r^{p}\right)_{x x x x}+\frac{n(n-1)}{2}\left(r^{p-2} r_{x}^{2}\right)_{x x}\right),
$$

while the latter establishes:

$$
r_{t t}=\left(r^{p}\right)_{x x}+\frac{\varepsilon^{2}}{12}\left(r^{p}\right)_{x x x x} .
$$



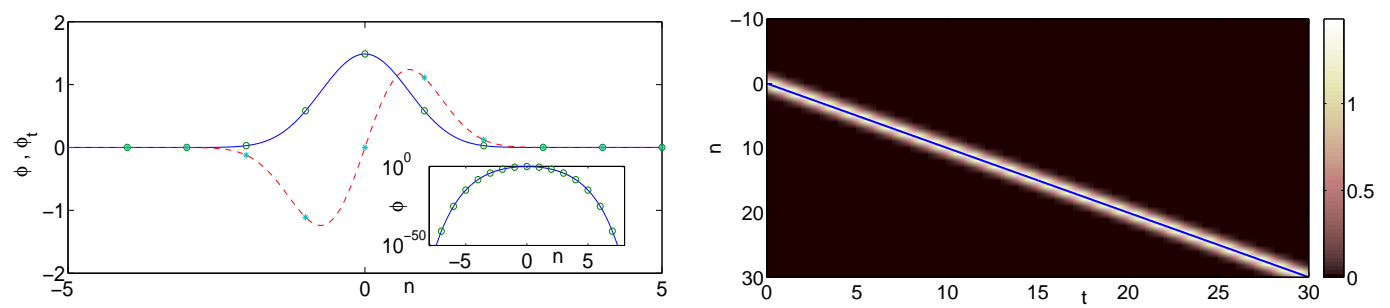

FIG. 3. (Color online) A typical example of the spatial profile of the positions (blue solid line connecting the lattice nodes) and of the velocities (red dashed line connecting the lattice nodes) of an exact (nearly-compact, see inset) traveling wave solution of the monomer granular chain, obtained through the solution of Eq. 5.3. The space $(n)$ - time $(t)$ evolution of this wave with its prescribed speed of unity is shown through the contour map of the strain $r_{n}(t)$.

In fact, from a mathematical perspective, one can go even further and suggest that since the above models are singularly perturbed (and may suffer relevant pathologies, which may also affect their computational implementation), it may be relevant to suggest the consideration of regularized variants of these PDEs, such as the one proposed by the work of Rosenau (1986) and Rosenau (2003) as:

$$
r_{t t}=\left(r^{p}\right)_{x x}+\frac{\varepsilon^{2}}{12} r_{x x t t}
$$

A systematic examination of the relevant models and a rigorous proof of the decay properties of its associated solutions (and how they connect to those of the discrete problem from which the approximation stems) would be a particularly interesting theme for future studies.

A recent direction of intense interest within this theme of granular crystals has concerned the examination of progressively more heterogeneous settings. In the work of Porter et al. (2008) and Porter et al. (2009), dimers of different types were considered (e.g., 1 steel bead-1 teflon bead, or 2 steel-1 teflon, $\ldots$, or 5 steel-1 teflon etc., as well as similarly with other materials such as brass, rubber, glass and nylon), as well as of trimers ( 1 steel bead, 1 brass bead, 1 teflon bead or 1 steel, 1 glass, 1 nylon, etc.). From a theoretical/mathematical perspective, one of the fundamental contributions of this work for the consideration of

$$
\begin{aligned}
& m_{1} \ddot{u}_{j}=\left(w_{j}-u_{j}\right)^{k}-\left(u_{j}-w_{j-1}\right)^{k}, \\
& m_{2} \ddot{w}_{j}=\left(u_{j+1}-w_{j}\right)^{k}-\left(w_{j}-u_{j}\right)^{k},
\end{aligned}
$$

(i.e., the reduced dimer granular setting) was the proposition of a modified Taylor expansion:

$$
w=\lambda\left(u+b_{1} D u_{x}+b_{2} D^{2} u_{x x}+b_{3} D^{3} u_{x x x}+b_{4} D^{4} u_{4 x}+\ldots\right) .
$$

This enabled the effective "homogenization" of the ordinary differential equations into a "dimer continuum medium", provided that the relevant consistency [between Eqs. (5.9) and (5.10)] conditions $\lambda=1$, $b_{1}=1, b_{2}=m_{1} /\left(m_{1}+m_{2}\right), b_{3}=\left(2 m_{1}-m_{2}\right) /\left(3\left(m_{1}+m_{2}\right)\right), b_{4}=m_{1}\left(m_{1}^{2}-m_{1} m_{2}+m_{2}^{2}\right) /\left(3\left(m_{1}+m_{2}\right)^{3}\right)$, etc. are satisfied. Notice that for $m_{1}=m_{2}$, these yield the regular Taylor expansion, as expected. This long-wavelength approach for the dimer problem produced a PDE of the type of Eq. 5.6), whose solutions were compared favorably to numerical computations and experimental results in the works of Porter et al. (2008) and Porter et al. (2009). 
On the other hand, the consideration of dimers enabled for the first time another possibility that has been very recently explored more systematically. In particular, in the presence of precompression, the dimer model becomes:

$$
m_{i} \ddot{u}_{i}=A\left[\delta_{0}+u_{i-1}-u_{i}\right]^{3 / 2}-A\left[\delta_{0}+u_{i}-u_{i+1}\right]^{3 / 2},
$$

where $m_{2 i+1}=M$ and $m_{2 i}=m$ for all $i \in Z$ (for same material, different radii $R_{i}$ beads in the simplest realization). Then, at the end of the first Brillouin zone, i.e., at $k=\frac{\pi}{2 \alpha}$, where $\alpha=R_{i}+R_{i+1}-\delta_{0}$ is the equilibrium distance between two adjacent beads, the linear spectrum possesses a gap between the upper cutoff of the acoustic branch, $\omega_{1}=\sqrt{2 K_{2} / M}$, and the lower cutoff of the optical one, $\omega_{2}=$ $\sqrt{2 K_{2} / m}$, where $K_{2}=\frac{3}{2} A \delta_{0}^{1 / 2}$. This gap and the corresponding reformulation of the granular chain as an approximate $K_{2}-K_{3}-K_{4}$ chain of the form:

$$
\begin{aligned}
m_{i} \ddot{u}_{i} & =K_{2}\left(u_{i+1}-2 u_{i}+u_{i-1}\right)+K_{3}\left(\left(u_{i+1}-u_{i}\right)^{2}-\left(u_{i-1}-u_{i}\right)^{2}\right) \\
& +K_{4}\left(\left(u_{i+1}-u_{i}\right)^{3}+\left(u_{i-1}-u_{i}\right)^{3}\right)
\end{aligned}
$$

with $K_{3}=-\frac{3}{8} A \delta_{0}^{-1 / 2}$ and $K_{4}=\frac{3}{48} A \delta_{0}^{-3 / 2}$ enables the adaptation of the findings of Huang \& $\mathrm{Hu}(1998)$, demonstrating that since $\frac{K_{3}^{2}}{K_{2} K_{4}}>\frac{3}{4}$, discrete gap breather modes will bifurcate from the optical band, in fact, due to the modulational instability of the band edge. The relevant nonlinear states were recently experimentally and numerically established in the work of Boechler et al. (2009). Furthermore, such breathing excitations were also established in the presence of impurities by Theocharis et al. (2009), as a nonlinear continuation of the linear modes induced by the presence of such defects. In that setting the relevant nonlinear impurity modes were obtained both in the setting of one defect, as well as in that of a defect "double well" (with two defects separated by one lattice node). Similar excitations but of a transient form, due to the absence of precompression (and hence of an underlying linear regime), were also recently observed experimentally (and discussed theoretically) in the work of Job et al. (2009).

It is interesting to also note that once the role of individual defects is starting to be explored, it is natural to examine the progressive inclusion of disorder within such granular chains. A few steps have been already taken in that direction. In particular, in the work of Ponson et al. (2009), a systematic approach towards increasing randomness within the lattice has been used, by examining a dimer setting, and progressively "flipping" the order of the masses in the dimer in order to create defects. In that case, a transition between the propagation of waves in low-disorder regimes and the degradation of the wave in high-disorder lattices was clearly illustrated. On the other hand, the incorporation of disorder can be used to achieve a degree of optimality in granular networks. An example in this direction is the work of Fraternali et al. (2010), where upon a given selection of masses and elastic properties thereof, a genetic algorithm approach was used towards the optimization (minimization) of the propagated force at the end of the chain. This was a first step towards the optimal construction of "granular protectors".

The above investigations are indicative of the intense recent activity in the field of granular crystals. Yet, there is a wide range of topics that require additional theoretical, numerical and experimental investigation. In the one-dimensional setting, in addition to exploring the existence (and stability) of traveling waves from a rigorous viewpoint not only in the monomer, but also in the "multimer" (i.e., dimer, trimer etc.) setting, it would also be of interest to explore more systematically the role of disorder. In particular, the setting of granular chains might be an ideal testbed where the recent developments of subdiffusive wavepacket spreading and possible "destruction" of Anderson localization in nonlinear lattices could be explored controllably. This is because this setting can be made, on demand, nearly linear, weakly or strongly (or even completely) nonlinear. Furthermore, while the above ideas discussed 
earlier based on the works of Kopidakis et al. (2008), Flach et al. (2009), Pikovsky \& Shepelyansky (2008) and Skokos et al. (2009), were established in the context of nonlinear models with onsite potentials such as the DNLS, it would be interesting to explore them in models within the FPU class i.e., with inter-site interactions, and especially ones such as the granular chain which could in principle be realized experimentally in a controllable fashion. On the other hand, another important observation in many of the above mentioned experimental works in granular chains [see also the relevant reviews of Nesterenko (2001) and Sen et al. (2008)] is the key role of dissipative perturbations in decreasing the amplitude of the propagating [or localized breathing as in Boechler et al. (2009)] waves. A widely applicable, quantitatively accurate modeling from first principles of such mechanisms is still missing despite relevant recent efforts and interesting corresponding results e.g. in the works of Rosas et al. (2007) and of Carretero et al. (2009). Another direction which still remains relatively pristine is that of higher dimensional structures. While some of the linear or near-linear behavior has been explored therein in both ordered and disordered granular chains, much less is known about the structure and propagation (as well as the localization) of nonlinear waves within such higher-dimensional granular crystals. This is a theme which, considering the activity and progress recently spurred in the one-dimensional setting, is likely to emerge as one of the most lively areas of investigation within the next decade.

Finally, we wanted to add a brief comment on FPU-type lattice applications in dusty plasmas and the potential emergence of nonlinear excitations in these settings. In recent works such as those of Koukouloyannis \& Kourakis (2007), Koukouloyannis \& Kourakis (2009) and Kourakis \& Shukla (2006), the setting of dusty plasmas has been proposed as a canonical realization of nonlinear Klein-Gordon lattices. The coupling between adjacent nodes/dust grains has been proposed to be linear, while the sheath environment has been argued to create a nonlinear onsite potential landscape that drives the relevant transverse dynamics considered in these works. In this context, both one- and two-dimensional discrete breathers of the type explored in the previous section for Klein-Gordon lattices have been identified and their stability has been accordingly examined. However, here, we would like to focus the attention instead on the broader context of e.g. the presentation of Kourakis \& Shukla (2006). There both longitudinal and transverse vibrations of the relevant dust particles were examined. Although the latter are, in principle, experimentally possible as in the work of Zafiu et al. (2001), there is also a particular interest in the former, as they incorporate the nonlinear potential of interaction across sites which can be accurately approximated by an exponentially dependent on the inter-particle separation Yukawatype landscape. Both the linear and the nonlinear implications of such lattices have been explored in some detail as e.g. in the recent review of Melzer et al. (2008). However, the emergence of nonlinear waveforms and especially the existence, stability and mobility of discrete breathers in these intersite but non-nearest-neighbor (in fact, the coupling is "all-to-all") lattices have received limited attention. We feel that this field is also presently at a ripe phase, where the use of the tools and technology of localized modes and their stability and dynamics could have a substantial impact in experimental, as well as numerical investigations.

\section{Instead of an Epilogue}

The field of nonlinear dynamical lattices has seen a tremendous explosion of interest associated with it over the past 20 years. Although the first anharmonic lattice investigations were initiated more than 50 years ago by Fermi, Pasta and Ulam, and part of these led to the remarkable theory of solitary waves and solitons which has been pervasive of various areas of science for more than the past 40 years, the appreciation of the idiosyncrasies of the lattices in comparison to their continuum siblings is far more recent. The seminal studies on intrinsically localized modes led to remarkable breakthroughs that fu- 
eled research in areas as diverse as nonlinear optics and optical waveguides, Bose-Einstein condensates in optical lattices, micromechanical systems, pendulum arrays, electrical lattices, DNA denaturation, Josephson junction arrays, split ring resonators and metamaterials, granular crystals, dusty plasmas, metallic uranium and ionic sodium iodide, and possibly even in sheets of graphene [see e.g., the recent work of Yamayose et al. (2007)]. Alongside the development of the areas of application, the particularities of each of these settings have forced not only a development of the general mathematical ideas and of fundamental conditions under which the relevant excitations should be expected to exist and be robustly preserved by the system dynamics, but have also inspired the more specific adaptation of these ideas and of the relevant modeling to the special settings of each particular application. They have also motivated the emergence of a diverse array of relevant excitations, ranging from solitons to discrete breathers, to multi-peaked structures or even to compactly (or nearly compactly) supported ones. It is clear that after the initial decade of enthusiasm and exploration, and the second one of broad and far reaching applicability, the field is now entering a new decade of maturity, but where significant challenges lie ahead. These concern the application of the relevant ideas to very recently emerging fields (materials and metamaterials, granular media, plasmas), their exploration in more realistic and especially higher dimensional settings and the establishment of more firm connections/interplays with other areas of physics, such as non-equilibrium thermodynamics, or the study of disordered media and of wave phenomena within them. What the resulting 20/20 vision of nonlinear waves in lattices will be remains to be seen...

Acknowledgments. This review would not have been possible without the help, collaboration and insights of numerous colleagues that have shaped my vision of the field through our interactions. As a partial list, Dimitri Frantzeskakis, Ricardo Carretero (who also contributed significantly towards improving this manuscript and is especially thanked for that), Boris Malomed, Yuri Kivshar, Volodya Konotop, Augusto Smerzi, Mario Salerno, Mason Porter, Chiara Daraio, Yannis Kourakis, Vassilis Koukouloyannis, George Theocharis, Jesus Cuevas, Dmitry Pelinovsky, Faustino Palmero, Wieslaw Krolikowski, Michael Weinstein, Chris Jones, Todd Kapitula, Shozo Takeno, Al Sievers, Alex Kovalev, Atanas Stefanov, Sergey Dmitriev, Alan Bishop, Avadh Saxena, Avinash Khare, Kim Rasmussen, Zhigang Chen, Kody Law, Andrea Trombettoni, Yuri Gaididei are especially thanked. The support of the National Science Foundation through the CAREER program (DMS-0349023) and through NSF-DMS0806762, as well as that of the Alexander von Humboldt Foundation through its research fellowship program are gratefully acknowledged.

\section{REFERENCES}

F. Kh. Abdullaev, B.B. Baizakov, S.A. Darmanyan, V.V. Konotop \& M. Salerno (2001) Nonlinear excitations in arrays of Bose-Einstein condensates, Phys. Rev. A 64, 043606.

M. ABlowitz \& J.F. LADiK (1976) Nonlinear differential-difference equations and Fourier analysis, J. Math. Phys., 17, 1011-1018.

M.J. Ablowitz, B. Prinari \& A.D. Trubatch (2004) Discrete and continuous nonlinear Schrödinger systems, Cambridge University Press, Cambridge.

K. Ahnert \& A. Pikovsky (2009) Compactons and chaos in strongly nonlinear lattices, Phys. Rev. E 79, 026209.

T. Ahn, R.S. MacKay \& J.-A. Sepulchre (2001) Dynamics of relative phases: generalised multibreathers, Nonlinear Dyn. 25 157-182.

T.J. Alexander, A.A. Sukhorukov \& Yu.S. Kivshar (2004) Asymmetric vortex solitons in nonlinear periodic lattices, Phys. Rev. Lett. 93, 063901.

G.L. Alfimov, P.G. Kevrekidis, V.V. Konotop \& M. Salerno (2002) Wannier functions analysis of the 
nonlinear Schrdinger equation with a periodic potential, Phys. Rev. E 66, 046608.

G.L. Alfimov, V.A. BraZhny \& V.V. Konotop (2004) On classification of intrinsic localized modes for the discrete nonlinear Schrödinger equation, Physica D 194, 127-150.

P.W. ANDERSON (1958) Absence of Diffusion in Certain Random Lattices, Physical Review 109, 1492-1505.

B.P. ANDERSON \& M.A. KASEVICH (1998) Macroscopic quantum interference from atomic tunnel arrays, Science 282, 1686-1689.

J.F.R. Archilla, J. Cuevas, B. SÁnchez-Rey \& A. Álvarez (2003) Demonstration of the stability or instability of multibreathers at low coupling, Physica D 180 235-255.

S. Aubry (1997) Breathers in nonlinear lattices: existence, linear stability and quantization, Physica D 103, 201250.

I.V. Barashenkov, O.F. OXtoby \& D.E. Pelinovsky (2005) Translationally invariant discrete kinks from one-dimensional maps, Phys. Rev. E 72, 035602.

I.V. Barashenkov \& T.C. VAn HeErden (2008) Exceptional discretizations of the sine-Gordon equation, Phys. Rev. E 77, 036601.

N. Boechler, G. Theocharis, S. Job, P.G. Kevrekidis, M.A. Porter, C. Daraio (2009) Discrete breathers in one-dimensional diatomic granular crystals, arXiv:0911.2817.

O.M. Braun \& YU.S. Kivshar (1998) Nonlinear dynamics of the Frenkel-Kontorova model, Phys. Rep. 306, $1-108$.

O.M. BRaun \& YU.S. Kivshar (2006) The Frenkel-Kontorova model: Concepts, Methods and Applications, Springer-Verlag, Berlin.

V.A. BraZhny \& V.V. Konotop (2004) Theory of nonlinear matter waves in optical lattices, Mod. Phys. Lett. B 18, 627-651.

S. Burger, F.S. Cataliotti, C. Fort, P. Maddaloni, F. Minardi \& M. Inguscio (2002) Quasi-2D BoseEinstein condensation in an optical lattice, Europhys. Lett. 57, 1-6.

F. CAlogero (1971) Solution of the One-Dimensional N-Body Problems with Quadratic and/or Inversely Quadratic Pair Potentials, J. Math. Phys., 12, 419-436.

D.M. Campbell, P. Rosenau \& G.M. Zaslavsky (2005) Introduction: The Fermi-Pasta-Ulam problem- The first fifty years, Chaos 15, 015101.

R. Carretero-González, P.G. Kevrekidis, B.A. Malomed \& D.J. Frantzeskakis (2005) ThreeDimensional Nonlinear Lattices: From Oblique Vortices and Octupoles to Discrete Diamonds and Vortex Cubes, Phys. Rev. Lett. 94, 203901.

R. Carretero-González, J.D. Talley, C. Chong \& B.A. Malomed (2006) Multistable solitons in the cubic-quintic discrete nonlinear Schrödinger equation, Physica D 216, 77-89.

R. Carretero-González, D. Khatri, M.A. Porter, P.G. Kevrekidis \& C. Daraio (2009) Dissipative Solitary Waves in Granular Crystals, Phys. Rev. Lett. 102, 024102.

F.S. Cataliotti, L. Fallani, F. Ferlaino, C. Fort, P. Maddaloni \& M. Inguscio (2003) Superfluid current disruption in a chain of weakly coupled Bose-Einstein condensates, New J. Phys. 5, 71.

Z. Chen, H. Martin, A. Bezryadina, D.N. Neshev, Yu.S. Kivshar \& D.N. Christodoulides (2005) Experiments on Gaussian beams and vortices in optically-induced photonic lattices, J. Opt. Soc. Am. B 22, 1395-1405.

C. Chong, R. Carretero-González, B.A. Malomed \& P.G. Kevrekidis (2009) Multistable solitons in higher-dimensional cubic-quintic nonlinear Schrödinger lattices, Physica D 238, 126-136.

P.L. Christiansen, Yu.B. Gaididei, M. Johansson, K.Ø. Rasmussen, V.K. Mezentsev \& J.J. RasMUSSEN (1998) Solitary excitations in discrete two-dimensional nonlinear Schrdinger models with dispersive dipole-dipole interactions, Phys. Rev. B 57, 11303-11318.

C. Coste, E. Falcon \& S. Fauve (1997) Solitary waves in a chain of beads under Hertz contact, Phys. Rev. E 56, 6104-6117.

J. Cuevas, L.Q. English, P.G. Kevrekidis \& M. Anderson (2009) Discrete Breathers in a Forced-Damped 
Array of Coupled Pendula: Modeling, Computation, and Experiment, Phys. Rev. Lett., 102, 224101.

C. Daraio, V.F. Nesterenko, E.B. Herbold \& S. Jin (2005) Strongly nonlinear waves in a chain of Teflon beads, Phys. Rev. E 72, 016603.

C. Daraio, V.F. Nesterenko, E.B. Herbold \& S. Jin (2006) Energy trapping and shock disintegration in a composite granular medium Phys. Rev. Lett. 96, 058002.

C. Daraio, V.F. Nesterenko, E.B. Herbold \& S. Jin (2006) Tunability of solitary wave properties in onedimensional strongly nonlinear phononic crystals, Phys. Rev. E 73, 026610.

C. Daraio, R. BAumer, D. Sun \& S. SEN (2009) Hertz-like laws and solitary waves in irregular bead chains (in progress).

A.S. DAVydov (1972) The theory of contraction of proteins under their excitation, J. Theoret. Biol. 38, 559-569.

A.S. Dolgov (1986) The localization of vibrations in a nonlinear crystal structure, Fizika Tverdogo Tela 28, 1641-1644.

R. Doney \& S. SEN (2006) Decorated, tapered, and highly nonlinear granular chain, Phys. Rev. Lett. 97, 155502.

D.B. Duncan, J.C. Eilbeck, H. Feddersen \& J.A.D. Wattis (1993) Solitons on lattices, Physica D 68, $1-11$.

N.K. Efremidis \& D.N. Christodoulides (2002) Discrete solitons in nonlinear zigzag optical waveguide arrays with tailored diffraction properties, Phys. Rev. E 65, 056607.

N.K. Efremidis, S. Sears, D.N. Christodoulides, J.W. Fleischer \& M. SegeV (2002) Discrete solitons in photorefractive optically induced photonic lattices, Phys. Rev. E, 66, 046602.

B. Eiermann, Th. Anker, M. Albiez, M. Taglieber, P. Treutlein, K.-P. Marzlin \& M.K. Oberthaler (2004) Bright Bose-Einstein gap solitons of atoms with repulsive interaction, Phys. Rev. Lett. 92, 230401.

J.C. Eilbeck \& M. Johansson (2003) The discrete nonlinear Schrödinger equation: 20 years on, Proceedings of the 3rd Conference on Localization \& Energy Transfer in Nonlinear Systems, World Scientific, New Jersey, 44-67.

J.C. Eilbeck, P.S. Lomdahl \& A.C. Scott (1985) The discrete self-trapping equation Physica D 16, 318-338.

H.S. Eisenberg, Y. Silberberg, R. Morandotti, A.R. Boyd \& J.S. Aitchison (1998) Discrete Spatial Optical Solitons in Waveguide Arrays, Phys. Rev. Lett. 81, 3383-3386.

M. Eleftheriou, N. Lazarides \& G.P. Tsironis (2009) Magnetoinductive breathers in metamaterials, Phys. Rev. $E$ 77, 036608.

L.Q. English, private communication.

J.M. ENGLish \& R.L. Pego (2005) On the solitary wave pulse in a chain of beads, Proceedings of the AMS 133, 1763-1768.

L.Q. English, M. Sato \& A.J. Sievers (2001) Nanoscale intrinsic localized modes in an antiferromagnetic lattice, J. Appl. Phys. 89, 6706-6709.

L.Q. English, M. SATo \& A.J. Sievers (2001) Modulational instability of nonlinear spin waves in easy-axis antiferromagnetic chains. II. Influence of sample shape on intrinsic localized modes and dynamic spin defects, Phys. Rev. B, 67, 024403 .

L.Q. English, R. Basu-Thakur \& R. Stearett (2008) Patterns of traveling intrinsic localized modes in a driven electrical lattice, Phys. Rev. E 77, 066601.

J.A. EsPinola-Rocha \& P.G. KeVREKIDIS (2009) Thresholds for soliton creation in the Ablowitz-Ladik lattice, Math. Comp. Simul. 80 693-705.

E. Fermi, J. Pasta \& S. Ulam (1954) Studies in Nonlinear Problems I, Los Alamos Report, 1940.

S. Flach \& A.V. Gorbach (2008) Discrete breathers - advances in theory and applications, Phys. Rep. 467, $1-116$.

S. Flach, M.V. Ivanchenko \& O.I. Kanakov (2005) q-Breather and the Fermi-Pasta-Ulam problem, Phys. Rev. Lett. 95, 064102.

S. FlaCh, D.O. KRIMER \& CH. SKOKOS (2009) Universal spreading of wave packets in disordered nonlinear systems, Phys. Rev. Lett. 102, 024101. 
S. Flach \& C.R. Willis (1998) Discrete Breathers, Phys. Rep. 295, 182-264.

J.W. Fleischer, M. SEgEv, N.K. Efremidis \& D.N. Christodoulides (2003) Observation of two-dimensional discrete solitons in optically induced nonlinear photonic lattices, Nature 422, 147-150.

J.W. Fleischer, G. Bartal, O. Cohen, O. Manela, M. Segev, J. Hudock \& D.N. Christodoulides (2004) Observation of vortex-ring "discrete" solitons in 2D photonic lattices, Phys. Rev. Lett. 92, 123904.

F. Fraternali, M.A. Porter \& C. Daraio (2010) Optimal design of composite granular protectors, Mech. Adv. Mat. Struct. 17, 1-19.

G. Galavotti (ed.) (2008) The Fermi-Pasta-Ulam problem: a status report, Springer-Verlag, Berlin.

B. Gilles \& C. Coste (2001) Nonlinear elasticity of a 2-D regular array of beads, Powders and Grains, Proceedings of the Fourth International Conference on Micromechanics of Granular Media, Sendai.

J.D. GodDARD (1990) Nonlinear elasticity and pressure-dependent wave speeds in granular media, Proceedings of the Royal Society A 430, 105-131.

C. Goldenberg \& I. Goldhirsch (2005) Friction enhances elasticity in granular solids, Nature, 435, $188-191$.

J. Gómez-Gardeñes, F. Falo \& L.M. Floría (2004) Mobile localization in nonlinear Schrödinger lattices, Phys. Lett. A 332, 213-219.

L. Hadzievski, A. Maluckov, M. Stepić \& D. Kip (2004) Power controlled soliton stability and steering in lattices with saturable nonlinearity, Phys. Rev. Lett. 93, 033901.

A.J. Heeger, S. Kivelson, J.R. Schrieffer \& W.-P. Su (1988) Solitons in conducting polymers, Rev. Mod. Phys., 60, 781-850.

D. Hennig \& G. Tsironis (1998) Wave transmission in nonlinear lattices, Phys. Rep. 307, 333-432.

J. Hong (2005) Universal power-law decay of the impulse energy in granular protectors, Phys. Rev. Lett. 94, 108001.

S.R. Hostler \& C.E. Brennen (2005) Pressure wave propagation in a granular bed, Physical Review E 72, 031303.

G. HUANG \& B. HU (1998) Asymmetric gap soliton modes in diatomic lattices with cubic and quartic nonlinearity, Phys. Rev. B 57, 5746-5757.

S. Job, F. Santibanez, F. TApia \& F. Melo (2009) Wave localization in strongly nonlinear Hertzian chains with mass defect, Phys. Rev. E 80, 025602.

M. JohANSSON \& K.Ø. RASMUSSEN (2004) Statistical mechanics of general discrete nonlinear Schrödinger models: localization transition and its relevance for Klein-Gordon lattices, Phys. Rev. E 70, 066610.

M. Johansson, Yu.B. Gaididei, P.L. Christiansen \& K.Ø. Rasmussen (1998) Switching between bistable states in a discrete nonlinear model with long-range dispersion, Phys. Rev. E 57, 4739-4742.

K.L. Johnson (2004) Contact Mechanics, Cambridge University Press, UK.

M. Jona-Lasinio, O. Morsch, M. Cristiani, N. Malossi, J.H. Müller, E. Courtade, M. Anderlini \& E. Arimondo (2003) Asymmetric Landau-Zener tunneling in a periodic potential, Phys. Rev. Lett. 91, 230406.

T. Kapitula, P.G. Kevrekidis \& B. SAndstede (2004) Counting eigenvalues via the Krein signature in infinite-dimensional Hamiltonian systems, Physica D 195, 263-282.

P.G. KeVREKIDIS (2003) On a class of discretizations of Hamiltonian nonlinear partial differential equations, Physica D 183, 68-86.

P.G. KEVREKIDIS (2009a) The discrete nonlinear Schroödinger equation, Springer-Verlag, Berlin.

P.G. KEVREKIDIS (2009b) Non-nearest-neighbor interactions in nonlinear dynamical lattices, Phys. Lett. A 372 3688-3693.

P.G. Kevrekidis \& D.E. Pelinovsky (2006) Discrete vector on-site vortices, Proc. Roy. Soc. A 462, 26712694.

P.G. Kevrekidis, K.Ø. Rasmussen \& A.R. Bishop (2001) The discrete nonlinear Schrödinger equation: a survey of recent results, Int. J. Mod. Phys. B 15, 2833-2900. 
P.G. Kevrekidis, B.A. Malomed, Z. Chen \& D.J. Frantzeskakis (2004a) Stable higher-order vortices and quasivortices in the discrete nonlinear Schrödinger equation, Phys. Rev. E 70, 056612.

P.G. Kevrekidis, B.A. Malomed, D.J. Frantzeskakis \& R. Carretero-GonzÁlez (2004b) ThreeDimensional Solitary Waves and Vortices in a Discrete Nonlinear Schrödinger Lattice, Phys. Rev. Lett. 93, 080403.

P.G. Kevrekidis, D.J. Frantzeskakis \& R. CArretero-GonzÁlez (eds.) (2008a) Emergent nonlinear phenomena in Bose-Einstein condensates, Springer-Verlag, Berlin.

P.G. Kevrekidis, J.A. Espinola-Rocha, Y. Drossinos \& A. Stefanov (2008b) Dynamical barrier for the formation of solitary waves in discrete lattices, Phys. Lett. A 372, 2247-2253.

P.G. Kevrekidis, G.J. Herring, S. Lafortune \& Q.E. Hoq (2009c) The higher-dimensional AblowitzLadik model: from (non-)integrability and solitary waves to surprising collapse properties and more exotic solutions, arXiv:0907.1386.

D. Khatri, C. DARAio \& P. Rizzo (2008) Coupling of Highly Nonlinear Waves with Linear Elastic Media, SPIE 6934, 69340U.

G. Kopidakis, S. Komineas, S. Flach \& S. Aubry (2008) Absence of Wave Packet Diffusion in Disordered Nonlinear Systems, Phys. Rev. Lett. 100, 084103.

A.M. Kosevich \& A.S. Kovalev (1974) Self-localization of vibrations in a one-dimensional anharmonic chain, Sov. Phys. JETP 67, 1793-1804.

V. Koukouloyannis \& P.G. KeVReKidis (2009) On the stability of multibreathers in Klein-Gordon chains, Nonlinearity 22 2269-2285.

V. Koukouloyannis \& I. KouraKis (2007) Existence of multisite intrinsic localized modes in one-dimensional Debye crystals, Phys. Rev. E 76, 016402.

V. Koukouloyannis \& I. Kourakis (2009) Discrete breathers in hexagonal dusty plasma lattices, Phys. Rev. E 80, 026402.

V. Koukouloyannis \& R.S. MACKAY (2005) Existence and stability of 3-site breathers in a triangular lattice, J. Phys. A: Math. Gen. 38, 1021-1030.

V. Koukouloyannis, P.G. KeVrekidis, K.J.H. Law, I. Kourakis \& D.J. Frantzeskakis (2009) Existence of discrete breathers in hexagonal and honeycomb lattices, arXiv:0906.2726.

I. KoURA KIS \& P.K. ShUKLA (2006) Nonlinear excitations in strongly-coupled plasma lattices: Envelope solitons, kinks and intrinsic localized modes, Int. J. Bif. Chaos 16, 1711-1725.

A. B. Kozyrev \& D. W. vAN DER WeIde (2008) Nonlinear left-handed transmission line metamaterials, J. Phys. D: Appl. Phys. 41, 173001.

D.O. KRIMER, R. KHOMERIKI \& S. FlaCh (2009) Delocalization and spreading in a nonlinear Stark ladder, Phys. Rev. E 80, 036201.

T. Lahaye, C. Menotti, L. Santos, M. Lewenstein \& T. Pfau (2009) The physics of dipolar bosonic quantum gases, Rep. Prog. Phys. 72, 126401.

Y. Lahini, A. Avidan, F. Pozzi, M. Sorel, R. Morandotti, D.N. Christodoulides \& Y. Silberberg (2008) Anderson Localization and Nonlinearity in One-Dimensional Disordered Photonic Lattices, Phys. Rev. Lett. 100, 013906.

R.F. LAMBERT \& J.S. TESAR (1984) Acoustic structure and propagation in highly porous, layered, fibrous materials, J. Acoust. Soc. Amer. 76, 1231-1237.

K.J.H. Law, P.G. Kevrekidis, V. Koukouloyannis, I. Kourakis, D.J. Frantzeskakis \& A.R. Bishop (2008) Discrete solitons and vortices in hexagonal and honeycomb lattices: Existence, stability, and dynamics, Phys. Rev. E 78, 066610.

K.J.H. Law, D. Song, P.G. Kevrekidis, J. XU \& Z. Chen (2009) Geometric stabilization of extended S=2 vortices in two-dimensional photonic lattices: theoretical analysis, numerical computation, and experimental results, Phys. Rev. A 80, 063817.

N. LAZARIDES, M. EleFTHERIOU \& G.P. TSIRONIS (2006) Discrete breathers in nonlinear magnetic metamate- 
rials, Phys. Rev. Lett. 97, 157406.

N. LAZARIDES, G.P. TSIRONIS \& YU.S. KivShaR (2008) Surface breathers in discrete magnetic metamaterials, Phys. Rev. E 77, 065601(R).

J.L. Lebowitz, H.A. Rose \& E.R. SPeER (1988) Statistical mechanics of the nonlinear Schrödinger equation, J. Stat. Phys. 50, 657-687.

F. Lederer, G.I. Stegeman, D.N. Christodoulides, G. Assanto, M. Segev \& Y. Silberberg (2008) Discrete solitons in optics Phys. Rep. 463, 1-126.

H. LeVy \& F. LeSsman (1992) Finite Difference Equations, Dover, New York.

N.M. Litchinitser \& V.M. ShalaeV (2008) Photonic metamaterials, Laser Phys. Lett. 5, 411-420.

R.S. MACKAY \& S. AUBRY, (1994) Proof of existence of breathers for time-reversible or Hamiltonian networks of weakly coupled oscillators, Nonlinearity 7, 1623-1643.

R.S. MacKay \& J.-A. Sepulchre (2002) Effective Hamiltonian for travelling discrete breathers, J. Phys. A 35 3985-4002.

B.A. Malomed \& P.G. Kevrekidis (2001) Discrete vortex solitons, Phys. Rev. E 64, 026601.

A. Maluckov, L. Hadzievski, N. Lazarides \& G.P. Tsironis, (2008) Left-handed metamaterials with saturable nonlinearity, Phys. Rev. E 77, 046607.

D. Mandelik, R. Morandotti, J.S. Aitchison \& Y. Silberberg (2004) Gap Solitons in Waveguide Arrays, Phys. Rev. Lett. 92, 093904.

R. MARqués, F. Martín \& M. Sorolla, John Wiley \& Sons, Hoboken.

H. Martin, E.D. Eugenieva, Z. Chen \& D.N. Christodoulides (2004) Discrete Solitons and SolitonInduced Dislocations in Partially Coherent Photonic Lattices, Phys. Rev. Lett. 92, 123902.

Joachim Meier, Jared Hudock, Demetrios Christodoulides, George Stegeman, Y. Silberberg, R. Morandotti \& J.S. Aitchison (2003) Discrete Vector Solitons in Kerr Nonlinear Waveguide Arrays, Phys. Rev. Lett. 91, 143907.

J. Meier, G.I. Stegeman, D.N. Christodoulides, Y. Silberberg, R. Morandotti, H. Yang, G. SAlamo, M. Sorel \& J.S. Aitchison (2004) Experimental Observation of Discrete Modulational Instability, Phys. Rev. Lett. 92, 163902.

T.R.O. Melvin, A.R. Champneys, P.G. Kevrekidis \& J. Cuevas (2006) Radiationless traveling qaves in saturable nonlinear Schrödinger lattices, Phys. Rev. Lett. 97, 124101.

T.R.O. Melvin, A.R. Champneys, P.G. Kevrekidis \& J. Cuevas (2008) Travelling solitary waves in the discrete Schrdinger equation with saturable nonlinearity: Existence, stability and dynamics, Physica D 237, 551-567.

M. Bonitz, P. Ludwig, H. Baumgartner, C. Henning, A. Filinov, D. Block, O. Arp, A. Piel, S. Käding, Y. IVAnov, A. Melzer, H. Fehske, V. Filinov (2008) Classical and quantum Coulomb crystals, Phys. Plasmas 15, 055704.

M.I. Molina, N. LAZARIDES, \& G.P. TsiRonis (2009) Bulk and surface magnetoinductive breathers in binary metamaterials, Phys. Rev. E 80, 046605.

R. Morandotti, U. Peschel, J.S. Aitchison, H.S. Eisenberg \& Y. Silberberg (1999) Dynamics of discrete solitons in optical waveguide arrays Phys. Rev. Lett. 83, 2726-2729.

R. Morandotti, H.S. Eisenberg, Y. Silberberg, M. Sorel \& J.S. Aitchison (1999) Self-Focusing and Defocusing in Waveguide Arrays, Phys. Rev. Lett. 86, 3296-3299.

O. Morsch \& M. Oberthaler (2006) Dynamics of Bose-Einstein condensates in optical lattices, Rev. Mod. Phys. 78, 179-215.

J. Moser (1975), Three integrable Hamiltonian systems connected with isospectral deformations, Adv. Math. 16, 197-220.

D. Neshev, E. Ostrovskaya, Yu.S. Kivshar \& W. Krolikowski (2003) Spatial solitons in optically induced gratings, Opt. Lett. 28, 710-712.

D. Neshev, Yu.S. Kivshar, H. Martin \& Z. Chen (2004) Soliton stripes in two-dimensional nonlinear 
photonic lattices, Opt. Lett. 29, 486-488.

D.N. Neshev, T.J. Alexander, E.A. Ostrovskaya, Yu.S. Kivshar, H. Martin, I. Makasyuk \& Z. CHEN (2004) Observation of discrete vortex solitons in optically induced photonic lattices, Phys. Rev. Lett. 92, 123903.

V.F. Nesterenko (2001) Dynamics of Heterogeneous Materials, Springer-Verlag, New York.

V.F. Nesterenko, C. Daraio, E.B. Herbold \& S. Jin (2005) Anomalous wave reflection at the interface of two strongly nonlinear granular media, Phys. Rev. Lett. 95, 158702.

M. ÖSTER \& M. JOHANSSON (2006) Stable stationary and quasiperiodic discrete vortex breathers with topological charge $\mathrm{S}=2$, Phys. Rev. E 73, 066608.

A.A. Ovchinikov (1970) Localized long-lived vibrational states in molecular crystals, Sov. Phys. JETP 30, 147.

O. Охтов \& I.V. BARAShenkov (2007) Moving solitons in the discrete nonlinear Schrödinger equation, Phys. Rev. E 76, 036603.

D.E. Pelinovs Ky (2006) Translationally invariant nonlinear Schrödinger lattices, Nonlinearity 19, 2695-2716.

D.E. Pelinovsky, P.G. KevReKidis \& D.J. FrantzeskaKis (2005a) Stability of discrete solitons in nonlinear Schrödinger lattices, Physica D 212, 1-19.

D.E. Pelinovsky, P.G. Kevrekidis \& D.J. Frantzeskakis (2005b) Persistence and stability of discrete vortices in nonlinear Schrödinger lattices, Physica D 212, 20-53.

M. Lukas, D.E. Pelinovsky \& P.G. Kevrekidis (2008) Lyapunov-Schmidt reduction algorithm for threedimensional discrete vortices, Physica D 237, 339-350.

M. PeYRARD (2004) Nonlinear dynamics and statistical physics of DNA, Nonlinearity 17, R1-R40.

A.S. Pikovsky \& D.L. Shepelyansky (2008) Destruction of Anderson Localization by a Weak Nonlinearity, Phys. Rev. Lett. 100, 094101.

L. Ponson, N. Boechler, Y.-M. Lai, M.A. Porter, P.G. Kevrekidis \& C. Daraio (2009) Elastic spin chains, arXiv:0904.0426.

M.A. Porter, C. Daraio, E.B. Herbold, I. Szelengowicz \& P.G. KevreKidis (2008) Highly nonlinear solitary waves in periodic dimer granular chains, Physical Review E 77, 015601(R).

M.A. Porter, C. Daraio, I. Szelengowicz, E.B. Herbold \& P.G. Kevrekidis (2009) Highly nonlinear solitary waves in heterogeneous periodic granular media, Physica D 238, 666-676.

K.Ø. Rasmussen, T. Cretegny, P.G. Kevrekidis \& N. Grønbech-Jensen Statistical mechanics of a discrete nonlinear system, Phys. Rev. Lett., 84, 3740-3743.

M. REMOISSENET (1999) Waves called solitons, Springer-Verlag, Berlin.

A. Rosas, A.H. Romero, V.F. Nesterenko \& K. Lindenberg (2007) Observation of Two-Wave Structure in Strongly Nonlinear Dissipative Granular Chains, Phys. Rev. Lett. 98, 164301.

P. Rosenau (1986) Dynamics of nonlinear mass-spring chains near the continuum limit, Phys. Lett. A 118, 222-227.

P. Rosenau (2003) Hamiltonian dynamics of dense chains and lattices: or how to correct the continuum, Phys. Lett. A 311, 39-52.

B. RUMPF (2004) Simple statistical explanation for the localization of energy in nonlinear lattices with two conserved quantities, Phys. Rev E 69, 016618.

L. Sanchez-Palencia, D. Clément, P. Lugan, P. Bouyer, G.V. Shlyapnikov \& A. Aspect (2007) Anderson Localization of Expanding Bose-Einstein Condensates in Random Potentials, Phys. Rev. Lett. 98, 210401.

M. Sato, B.E. Hubbard \& A.J. Sievers, (2006) Colloquium: Nonlinear energy localization and its manipulation in micromechanical oscillator arrays, Rev. Mod. Phys. 78, 137-157.

M. Sato, S. Yasui, M. Kimura, T. Hikihara \& A.J. Sievers (2007) Management of localized energy in discrete nonlinear transmission lines, Europhys. Lett. 80, 30002.

T. SCH ̈̈FFER \& C.E. WAYNE (2004) Propagation of ultra-short optical pulses in cubic nonlinear media, Physica D 196, 90-105. 
T. Schwartz, G. Bartal, S. Fishman \& M. Segev (2007) Transport and Anderson localization in disordered two-dimensional photonic lattices, Nature 446, 52-55.

S. Sen, J. Hong, J. BAng, E. Ávalos \& R. Doney (2008) Solitary waves in the granular chain, Phys. Rep. 462, 21-66.

A.J. Sievers \& S. Takeno, (1988) Intrinsic Localized Modes in Anharmonic Crystals, Phys. Rev. Lett., 61, 970-973.

Ch. Skokos, D.O. Krimer, S. Komineas \& S. Flach (2009) Delocalization of wave packets in disordered nonlinear chains, Phys. Rev. E 79, 056211.

A. Smerzi, A. Trombettoni, P.G. Kevrekidis \& A.R. Bishop (2002) Dynamical superfluid-insulator transition in a chain of weakly coupled Bose-Einstein condensates, Phys. Rev. Lett. 89, 170402.

J.M. SPEIGHT (1999) Topological discrete kinks, Nonlinearity 12, 1373-1387.

J.M. SPEIGHT \& R.S. WARD (1994) Kink dynamics in a novel discrete sine-Gordon system, Nonlinearity 7, 475-484.

C. Sulem \& P.L. Sulem (1999) The Nonlinear Schrödinger Equation, Springer-Verlag, New York.

S. Suntsov, K.G. Makris, D.N. Christodoulides, G.I. Stegeman, A. Haché, R. Morandotti, H. YAng, G. SAlamo \& M. Sorel (2006) Observation of Discrete Surface Solitons Phys. Rev. Lett. 96, 063901.

W.-P. Su, J.R. Schrieffer \& A.J. HeEger, (1979) Solitons in Polyacetylene, Phys. Rev. Lett., 42, 1698-1701.

B.I. Swanson, J.A. Brozik, S.P. Love, G.F. Strouse, A.P. Shreve, A.R. Bishop, W.-Z. Wang \& M.I. SALKOLA (1999) Observation of Intrinsically Localized Modes in a Discrete Low-Dimensional Material, Phys. Rev. Lett., 82, 3288-3291.

A. Szameit, T. Pertsch, S. Nolte, A. Tunnermann \& F. Lederer (2008) Long-range interaction in waveguide lattices, Phys. Rev. A 77, 043804.

S. TAKeno, K. Kisoda \& A.J. Sievers (1988) Intrinsic localized vibrational modes in anharmonic crystals, Prog. Theor. Phys. Suppl. 94, 242-269.

B. Terhalle, T. Richter, K.J.H. Law, D. Göries, P. Rose, T.J. Alexander, P.G. Kevrekidis, A.S. Desyatnikov, W. Krolikowski, F. Kaiser, C. Denz \& Yu.S. Kivshar (2009) Observation of doublecharge discrete vortex solitons in hexagonal photonic lattices, Phys. Rev. A 79, 043821.

G. Theocharis, M. Kavousanakis, P.G. Kevrekidis, C. Daraio, Mason A. Porter \& I.G. KevreKidis (2009) Localized breathing modes in granular crystals with defects, Phys. Rev. E 80, 066601.

M. TODA (1981), Theory of Nonlinear Lattices, Spinger-Verlag (Berlin).

A. Trombettoni \& A. Smerzi (2001) Discrete Solitons and Breathers with Dilute Bose-Einstein Condensates, Phys. Rev. Lett. 86, 2353-2356.

N.L. Tsitsas, T.P. Horikis, Y. Shen, P.G. KeVReKidis, N. Whitaker \& D.J. Frantzeskakis (2010) Short pulse equations and localized structures in frequency band gaps of nonlinear metamaterials, Phys. Lett. A 374, 1384-1388.

A.F. Vakakis, O. Gendelman, L. Bergman, D.M. McFarland, G. Kerschen \& Y.S. LeE (2008) Nonlinear Targeted Energy Transfer in Mechanical and Structural Systems, Springer-Verlag, Berlin.

X. WANG, Z. ChEN \& P.G. KeVReKIDIS (2006) Observation of discrete solitons and soliton rotation in optically induced periodic ring lattices, Phys. Rev. Lett. 96, 083904.

X. Wang, A. Bezryadina, Z. Chen, K.G. Makris, D.N. Christodoulides \& G.I. Stegeman (2007) Observation of two-dimensional surface solitons, Phys. Rev. Lett. 98, 123903.

Y. Yamayose, Y. Kinoshita, Y. Doi, A. NAKatani \& T. Kitamura (2007) Excitation of intrinsic localized modes in a graphene sheet, Europhys. Lett. 80, 40008.

J. YANG, I. MAKASYUK, A. BEZRYADINA \& Z. CHEN (2004) Dipole solitons in optically induced two-dimensional photonic lattices, Opt. Lett. 29, 1662-1664 (2004).

J. YAng, I. MAKASYUK, A. BEZRYAdina \& Z. Chen (2004) Dipole and quadrupole solitons in optically induced two-dimensional photonic lattices: theory and experiment, Stud. Appl. Math. 113, 389-412. 
J. Yang, I. Makasyuk, P.G. Kevrekidis, H. Martin, B.A. Malomed, D.J. Frantzeskakis \& ZhiGANG CHEN (2005) Necklacelike solitons in optically induced photonic lattices, Phys. Rev. Lett., 94, 113902.

N.J. ZABUSKY \& M. KRUSKAL, (1967) Interaction of "solitons" in a collisionless plasma and the recurrence of initial states, Phys. Rev. Lett., 15, 240-243.

C. ZafiU, A. Melzer \& A. Piel (2001) Nonlinear resonances of particles in a dusty plasma sheath Phys. Rev. E 63, 066403. J.W. Fleischer, G. Bartal, O. Cohen, T. Schwartz, O. Manela, B. Freedman, M. Segev, H. Buljan \& N.K. Efremidis (2005) Spatial photonics in nonlinear waveguide arrays Opt. Express 13, 1780-1796. 